\title{
Robots cuspidaux : théorie et applications
}

\section{Cuspidal robots: theory and applications}

\author{
par Philippe WENGER
}

Directeur de Rercherche CNRS

Laboratoire des Sciences du Numérique de Nantes, UMR CNRS 6004, Nantes, France

par Damien CHABLAT

\author{
Directeur de Rercherche CNRS
}

Laboratoire des Sciences du Numérique de Nantes, UMR CNRS 6004, Nantes, France

\section{Résumé}

Les robots étudiés dans cet article sont à base fixe et déplaçant un outil à l'aide d'une ou plusieurs chaînes articulées reliée à leur base. Les robots cuspidaux ont la capacité de changer de posture ou de mode d'assemblage sans jamais franchir une singularité. Ces robots sont longtemps restés méconnus alors qu'ils sont les plus nombreux. Cet article traite des robots cuspidaux sous des aspects tant fondamentaux qu'applicatifs. Il aborde les questions importantes soulevées par ces robots pour la conception et la planification de trajectoires. L'identification de tous les robots cuspidaux reste encore une question ouverte. L'article propose une classification complète d'une famille de robots sériels à trois articulations et aborde le cas des robots d'architectures plus complexes comme les robots parallèles.

\begin{abstract}
The robots studied in this article are fixed-base manipulators moving a tool through one or more articulated chains connected to their base. Cuspidal robots can change their posture or assembly mode without ever passing through a singularity. For a long time, these robots have remained unknown even though they are more numerous. This article deals with the fundamental and application aspects of cuspidal robots. It addresses the important issues raised by these robots for the design and planning of trajectories. The identification of all cuspidal robots is still an open issue. The paper proposes a complete classification of serial robots with three joints and addresses the case of robots with more complex architectures such as parallel robots.
\end{abstract}

\section{Mots-clés}

Robots sériels, Robots parallèles, Singularités, Planification de trajectoire.

\section{Keywords}

Serial robots, Parallel robots, Singularities, Trajectory planning. 


\section{Table des matières}

1 Préliminaires

1.1 Types de robots étudiés . . . . . . . . 3

1.2 Postures et modes d'assemblage . . . . 3

1.3 Singularités . . . . . . . . . . . . . 3

1.4 Aspects . . . . . . . . . . . . . 4

2 Robot cuspidal : définition, identification et propriétés

2.1 Définition et exemple . . . . . . . . 5

2.2 Changement de posture singulier et non singulier

2.3 Identification des robots cuspidaux .

3 Faisabilité de trajectoires

3.1 Régions t-parcourables pour un robot non cuspidal . . . . . . . . . .

3.2 Régions t-parcourables pour un robot cuspidal . . . . . . . . . . . 9

3.3 Influence des limites articulaires . . . . 10

4 Enumération et classification des robots cuspidaux et non-cuspidaux

4.1 Conditions géométriques simples conduisant à des robots non-cuspidaux . . . .

4.2 Classification des robots $3 R$ orthogonaux

4.3 Condition nécessaire et suffisante de cuspidalité d'un robot $3 R$ orthogonal . . . . 12

4.4 Cas des robots à 6 articulations . . . . . 12

4.4.1 Robots à poignet rotule . . . . . 12

4.4.2 Robots sans poignet rotule . . . 14

4.5 Règles de conception d'un robot . . . . 15

5 Étude de quelques robots parallèles cuspidaux

5.1 Les aspects pour les robots parallèles . . 16

5.2 Robot parallèle cuspidal . . . . . . . . 16

5.3 Identifier les robots parallèles cuspidaux 18

5.4 Classification des robots parallèles . . . 18

5.5 Faisabilité de trajectoires pour les robots parallèles . . . . . . . . . . . 20

5.5.1 Impact des changements de mode d'assemblage . . . . . 20

5.5.2 Taille des régions t-parcourables 22

5.6 Cas des robots à 6 degrés de liberté .. 23

5.7 Cas d'un robot parallèle cuspidal sans point cusp ... . . . . . . . . . 24

6 Conclusion la possibilité d'atteindre une cible de leur espace de travail selon plusieurs postures, par exemple avec les postures "coude haut" et "coude bas". Un changement de posture peut être opéré pour éviter une limite articulaire sur un axe ou une collision avec un obstacle. II a longtemps été admis que le robot devait alors nécessairement franchir une configuration singulière au cours d'un changement de posture, à l'image de ce qui se passe pour la plupart des robots industriels : la configuration singulière "bras tendu" doit toujours être franchie pour passer entre les postures "coude haut" et "coude bas". Si cette propriété est effectivement observée sur la plupart des robots qui ont des particularités géométriques (axes parallèles ou concourants), elle s'avère fausse pour de nombreux autres robots. Dès lors, plusieurs questions cruciales se posent, tant pour l'utilisateur que pour le concepteur. Comment savoir si le robot a bien cette propriété? Si ce n'est pas de cas, comment savoir si le robot a changé de posture? Quels sont les robots qui ont cette propriété?

Cet article se propose de répondre à ces questions. Un robot cuspidal est défini comme un robot qui peut changer de posture sans franchir une configuration singulière. La plus grande part de cet article traite des robots de type sériel. Il propose une méthodologie permettant d'identifier les robots cuspidaux et décrit de façon approfondie leur mécanisme de changement de posture. Une attention plus particulière est accordée à une famille de robots à trois articulations pivot d'axes orthogonaux deux à deux. Ces robots peuvent constituer des alternatives intéressantes aux robot usuels. II est donc important de pouvoir les classifier en robots cuspidaux et non cuspidaux. Les robots à six articulations sont plus difficiles à analyser et font encore l'objet de travaux de recherche au moment de la rédaction de cet article. Ils sont principalement abordés au travers de quelques exemples de robots $6 R$ cuspidaux, dont certains sont utilisés dans l'industrie comme robots de peinture. Le cas des robots d'architecture parallèle, plus délicat, est également abordé. On analyse des exemples de robots parallèles plans, sphériques et à 6 degrés de liberté, capables de changer de mode d'assemblage sans franchir une singularité. $L$ 'article propose enfin une discussion sur l'intérêt des robots cuspidaux.

\section{Introduction}

Lorsqu'un nouveau robot doit être implanté dans un site de production, il doit faire l'objet d'un choix initial de son architecture cinématique (sérielle ou parallèle, choix du nombre et des types d'axes ...) et, dans un second temps, il doit être programmé et piloté afin que l'outil qu'il manipule puisse suivre convenablement les trajectoires de consigne qui auront été définies pour réaliser les tâches demandées. La plupart des robots sériels ont 


\section{Bref historique des robots cuspidaux}

La toute première mention d'un changement de posture non singulier remonte à 1988 par deux chercheurs de l'Université de Bologne [PI88] qui mettent en évidence ce phénomène de façon numérique sur deux robots sériels différents à 6 articulations pivot. Cette révélation passa inaperçue et fut même parfois considérée comme fantaisiste. II faut dire qu'à l'époque, la communauté était convaincue que tout robot devait nécessairement franchir une singularité pour changer de posture, comme c'était le cas pour tous les robots industriels. Deux ans auparavant, une preuve mathématique - qui se révéla fausse par la suite- avait même été produite pour confirmer cette hypothèse [BL86]. Peu après la révélation des chercheurs de Bologne, J.W. Burdick (Université de Stanford, Californie) confirma les révélations des italiens dans sa thèse de doctorat, cette fois sur plusieurs robots sériels à 3 articulations pivot. Ce n'est qu'en 1992 qu'un nouveau formalisme est proposé pour les robots sériels capables de changer de posture sans passer par une singularité [Wen92], en pointant une erreur dans la démonstration qui avait été produite dans [BL86]. II a fallu de nombreuses années avant que la communauté scientifique ne commence à accepter l'existence de robots cuspidaux. Le terme robot cuspidal a été introduit en 1995 avec la démonstration de l'existence d'un point "cusp" (voir figure 7) sur le lieu des singularités des robots sériels cuspidaux à 3 articulations pivot [EW95]. Finalement, une classification exhaustive des robots sériels à 3 articulations pivots d'axes orthogonaux a été réalisée en 2004 [BWC04]. Un article de synthèse sur les robots sériel cuspidaux a été publié dans [Wen07] Les études sur les robot parallèles cuspidaux ont été initiées en 1998 [CW98] et sont majoritairement limitées aux robots plans.

\section{Préliminaires}

\subsection{Types de robots étudiés}

Deux grandes catégories de robots sont étudiées dans cet article. La première regroupe les robots sériels, dont l'organe terminal (un outil ou une pince) est relié à une base fixe par l'intermédiaire d'une seule chaîne cinématique articulée (Figure 1, gauche). La seconde catégorie, les robots parallèles [CB15], déplacent une plate-forme portant l'organe terminal par l'intermédiaire de plusieurs chaînes cinématiques articulées montées en parallèle (Figure 1, droite). La plus grande partie de l'article (chapitres 2, 3 et 4 ) sera consacrée aux robots sériels, et plus particulièrement aux robots de type $3 R$, c'est-à-dire dont la chaîne articulée est composée de trois articulations de type pivot (ou rotoïde, "revolute" en anglais, d'où la lettre "R") comme sur la figure 1 à gauche. Enfin, tous les robots étudiés dans cet article sont nonredondants, c'est-à-dire que leur nombre degrés de libertés (leur nombre d'articulations motorisées) est égal au nombre de coordonnées décrivant la tâche. C'est le cas des deux robots à 3 degrés de liberté de la figure 1 où la tâche est définie par trois coordonnées de position dans l'espace.

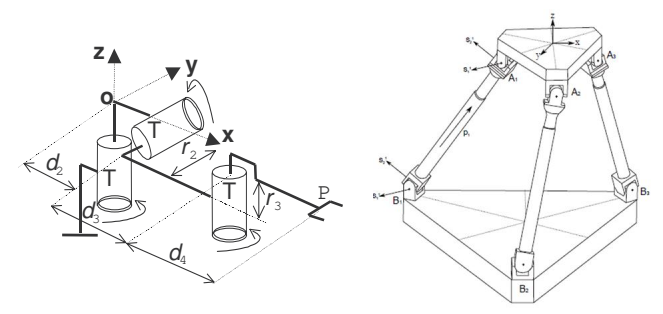

Figure 1 - Robot sériel de type 3R (à gauche), robot parallèle à 3 jambes télescopiques (à droite).

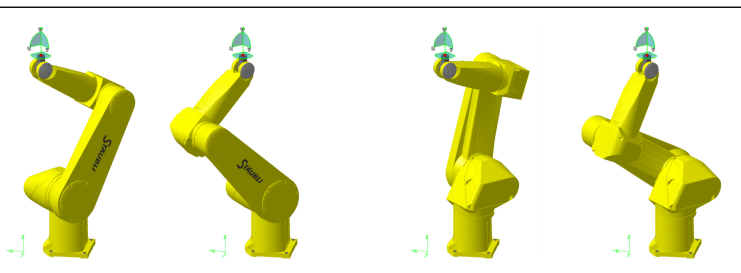

Figure 2 - Quatre postures d'un robot anthropomorphe identifiables par la position du coude et de l'épaule.

\subsection{Postures et modes d'assemblage}

Posture : pour un robot sériel, le terme posture définit une configuration articulaire permettant à l'organe terminal du robot d'atteindre une cible donnée dans son espace de travail. Une posture est donc associée à une solution au modèle géométrique inverse.

Pour les robots industriels courants, une posture peut être facilement identifiée. Pour un robot anthropomorphe tel que celui de la figure 2, par exemple, ces postures peuvent être identifiées par la position du coude (haut ou bas), de l'épaule (droite ou gauche) et du poignet (pronation ou supination). Le nombre total de combinaisons s'élève à $2^{3}=8$ postures distinctes. La figure 2 montre les quatre postures qui peuvent être identifiées par la position du coude et de l'épaule.

Mode d'assemblage : pour un robot parallèle, le terme mode d'assemblage définit une solution au modèle géométrique direct, c'est-à-dire une position et/ou orientation de la plate-forme mobile du robot correspondant aux variables articulaires motorisées.

La figure 3 montre les six modes d'assemblage d'un robot parallèle plan à trois jambes télescopiques. Pour un jeu de longueurs données des jambes $(A i, B i)$, la plateforme triangulaire $(B 1, B 2, B 3)$ peut prendre six positions et orientations différentes dans le plan.

\subsection{Singularités}

On rappelle brièvement ici le concept de singularité, leur rôle étant essentiel pour les robots cuspidaux. Une singularité d'un robot sériel définit une configuration articulaire à partir de laquelle le robot ne peut plus produire certains déplacements. De plus, au moins deux solutions géométriques inverses coïncident lorsque le robot est sur une singularité. La figure 4 en haut montre un robot an- 


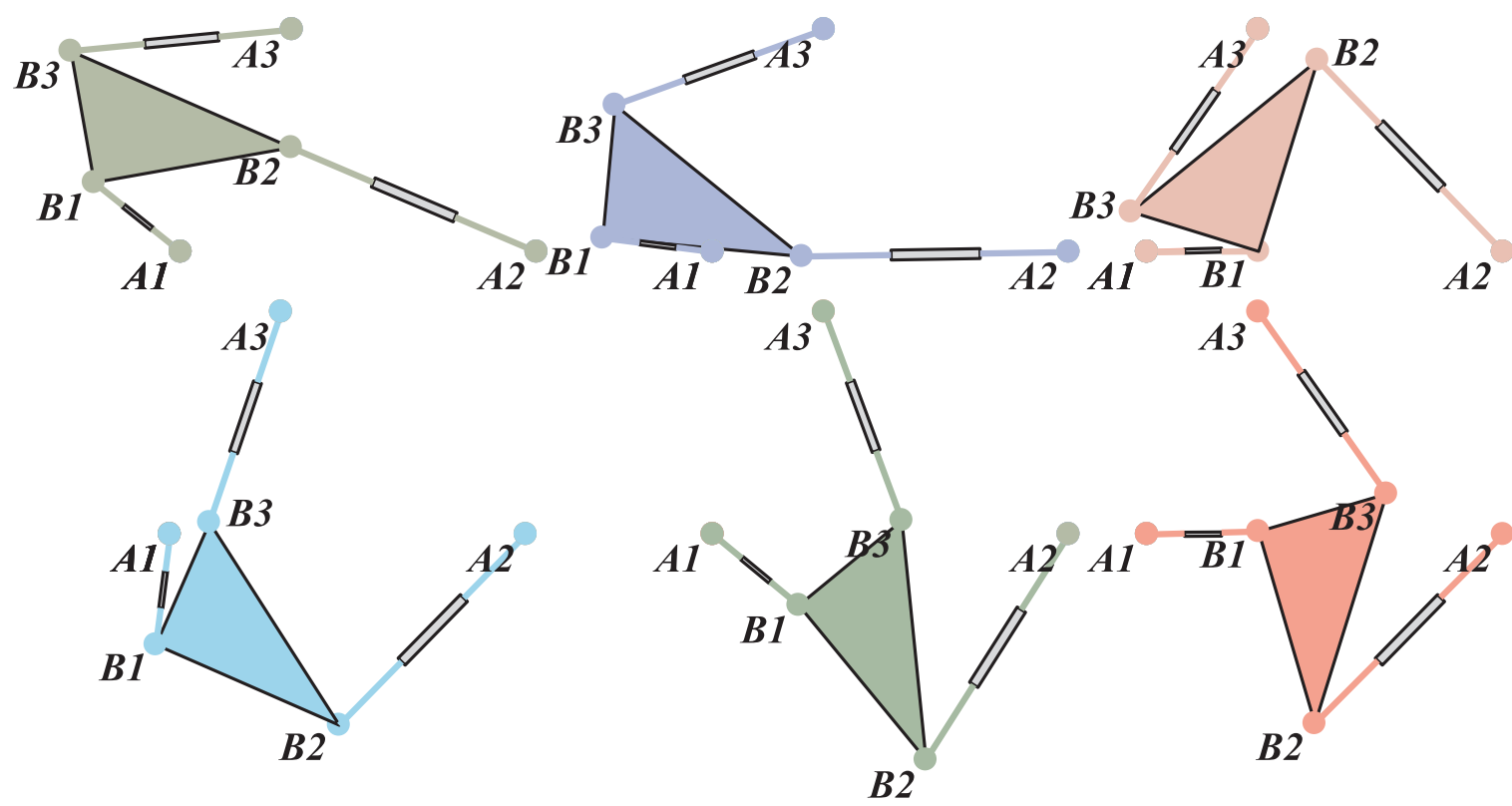

Figure 3 - Les six modes d'assemblage d'un robot parallèle plan à trois jambes télescopiques.

thropomorphe (représenté sans son poignet) en singularité de type "bras tendu" : localement, un déplacement le long de la direction du bras n'est alors pas possible. Sur cette singularité, les deux solutions inverses associées aux postures "coude haut" et "coude bas" montrées en bas de la figure 4 coïncident. Les singularités génèrent des frontières dans l'espace de travail qui ne peuvent généralement pas être franchies, car situées au bord de l'espace de travail. La singularité "bras tendu", par exemple, définit la limite d'accessibilité du robot.

On voit que pour le robot de la figure 4, la transition entre les deux solutions coude haut et coude bas se fait nécessairement en traversant la singularité bras tendu.

Les robots parallèles peuvent avoir plusieurs types de singularité. Dans cet article, on ne considérera que des robots parallèles avec des singularités de "type 2", également appelées "singularités parallèles" [CB15]. Sur ces singularités, le robot parallèle a certains mouvements qui ne peuvent plus être contrôlés. De plus, au moins deux de ses modes d'assemblage coïncident.

\subsection{Aspects}

Les aspects ont été définis initialement pour les robots sériels par Borrel [BL86]. On rappelle la définition ci-après pour les robots non-redondants.

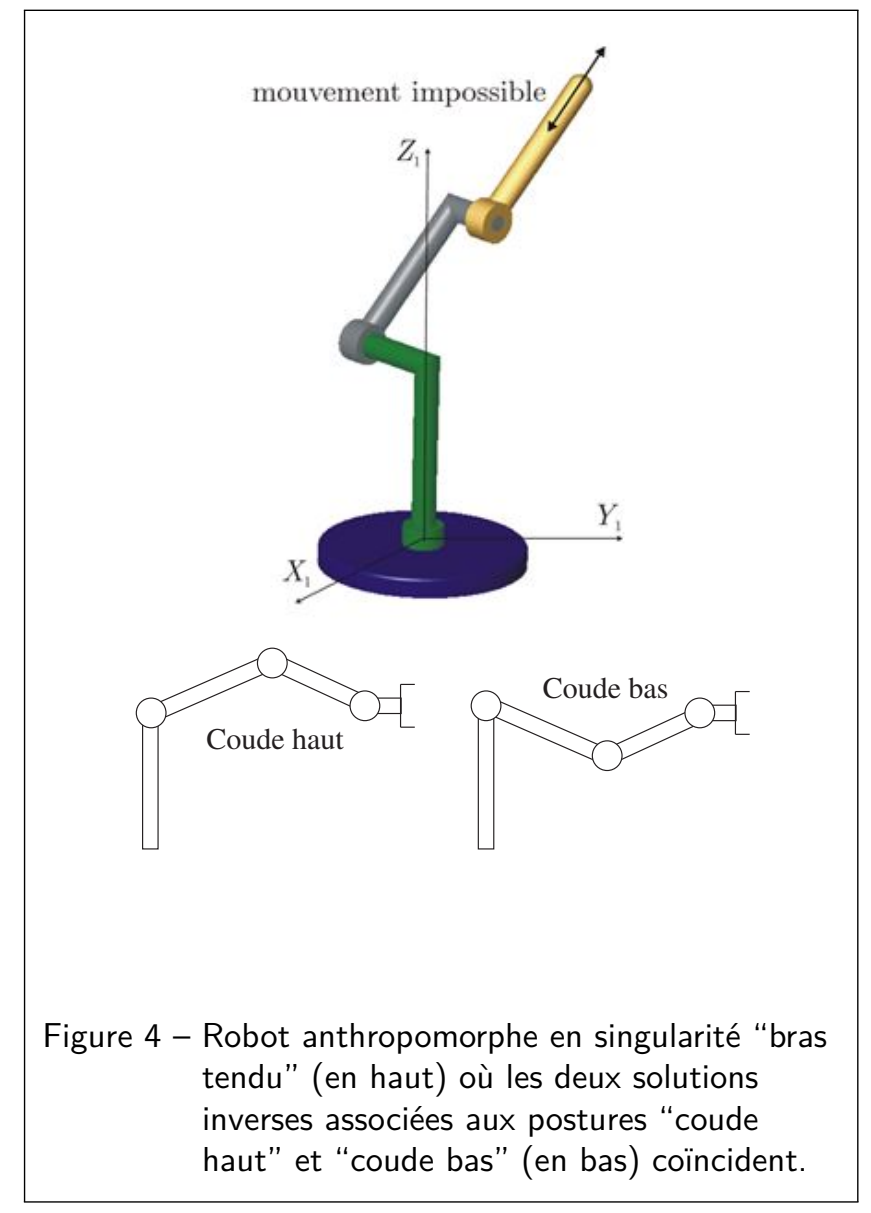


Les aspects pour les robots sériels non-redondants Soit $\mathcal{D}$ le domaine articulaire accessible:

$$
\mathcal{D}=\left\{\mathbf{q} \mid q_{\text {imin }} \leq q_{i} \leq q_{\text {imax }}, \forall i \in[i, n]\right\}
$$

où $\mathbf{q}=\left[q_{1}, . ., q_{n}\right]$ est le vecteur des $n$ variables articulaires des articulations motorisées.

Soit $\mathbf{X}=\left[x_{1}, \ldots, x_{n}\right]$ un choix de coordonnées opérationnelles définissant la pose de l'organe terminal. Ces coordonnées sont reliées aux variables articulaires par l'opérateur géométrique $\mathbf{f}$ défini par $\mathbf{X}=\mathbf{f}(\mathbf{q})$, soit $x_{i}=f_{i}(\mathbf{q}), \mathbf{i}=\mathbf{1}, . ., \mathbf{n}$.

On appelle aspects, les domaines $\mathcal{A}_{j}$ de $\mathcal{D}$ tels que

- $\mathcal{A}_{j}$ est connexe

- $\forall \mathbf{q} \in \mathcal{A}_{\mathbf{j}}, \operatorname{det}(\mathbf{J}) \neq 0$ où $\mathbf{J}=\left[\frac{\partial f_{i}}{\partial q_{i}}(\mathbf{q})\right]$ est la matrice jacobienne du robot.

Les aspects sont délimités par les singularités et les limites articulaires lorsqu'elles existent. Pour les robots sériels non redondants, les aspects ainsi définis sont les plus grands domaines de $\mathcal{D}$ exempts de toute singularité. Pour la plupart des robots industriels (et plus précisément comme on le verra plus tard, pour tous les robots non cuspidaux), les aspects constituent les domaines d'unicité de l'opérateur géométrique $\mathbf{f}$, c'est-à-dire qu'il n'y a qu'une seule solution inverse dans chaque aspect. Les aspects ont été généralisés par la suite aux robots parallèles [Cha98].

\section{Exemple 1 : Aspects d'un robot 3R}

Soit un robot de type $3 \mathrm{R}$ comme sur la Figure 1 à gauche, dont les paramètres géométriques sont : $d_{2}=1, d_{3}=2, d_{4}=1.5, r_{2}=1, r_{3}=0$, $\alpha_{2}=-90^{\circ}$ et $\alpha_{3}=90^{\circ}$. Les dimensions sont données sans précision d'unité, sans importance pour la compréhension de l'exemple. Ce robot est dit orthogonal car ses axes sont orthogonaux deux à deux. On suppose de plus que le robot n'a pas de limites articulaires. Ce robot sera utilisé dans la plupart des exemples tout au long de cet article. On montre que le déterminant de la matrice jacobienne $\mathbf{J}$ de ce robot peut s'écrire sous la forme d'un produit de deux facteurs (voir par exemple [El 96]) :

$$
\begin{aligned}
& \operatorname{det}(\mathbf{J})=\left(d_{3}+\cos \left(\theta_{3}\right) d_{4}\right) \\
& \left(\cos \left(\theta_{2}\right)\left(\sin \left(\theta_{3}\right) d_{3}-\cos \left(\theta_{3}\right) r_{2}\right)+\sin \left(\theta_{3}\right) d_{2}\right)
\end{aligned}
$$

On voit que les singularités, définies $\operatorname{par} \operatorname{det}(\mathbf{J})=$ 0 , ne dépendent pas de $\theta_{1}$, ce qui est toujours le cas lorsque la première articulation est de type pivot. On peut donc représenter l'espace articulaire dans le plan $\left(\theta_{2}, \theta_{3}\right)$. Le premier facteur $\operatorname{de} \operatorname{det}(\mathbf{J})$ ne peut pas s'annuler ici car $d_{4}<d_{3}$. Le second facteur définit deux courbes de forme identique et translatées l'une par rapport à l'autre de $\pi$ selon l'axe des $\theta_{3}$. Les singularités divisent alors le domaine articulaire en deux aspects (en vert et en bleu sur la figure 5 à gauche). En effet, comme il n'y a pas de limites articulaires, il convient d'iden- tifier les cotés opposés du domaine articulaire. Dans l'espace de travail qui est de dimension 3, les singularités définissent des surfaces frontières. Comme les singularités ne dépendent pas de $\theta_{1}$ et qu'il n'y a pas de limite articulaire, l'espace de travail est symétrique autour de l'axe 1 du robot. II est donc suffisant de n'en représenter qu'une demisection dans un plan passant par cet axe. Une telle section, appelée section génératrice, peut être définie dans le plan $(\rho, z)$ où $\rho=\sqrt{x^{2}+y^{2}}$. L'espace de travail total peut être obtenu en faisant tourner cette section de $360^{\circ}$ autour de l'axe 1 du robot. Pour le robot étudié, les singularités génèrent deux courbes fermées dans la section génératrice de l'espace de travail. L'une définit la frontière extérieure, l'autre marque une frontière intérieure entre une zone centrale accessible avec 4 postures et une zone périphérique accessible avec 2 postures (figure 5 à droite).

A retenir : Les robots sériels (resp. parallèles) possèdent plusieurs solutions géométriques inverses appelés postures (resp. plusieurs solutions géométriques directes appelées modes d'assemblage). Les singularités sont d'une importance fondamentale pour l'analyse du comportement des robots. Outre leur effet sur les déplacements possibles, elles divisent le domaine articulaire des robots sériels en domaines sans singularité appelés aspects.

\section{Robot cuspidal : définition, identification et propriétés}

\subsection{Définition et exemple}

Robot cuspidal : Un robot est dit cuspidal s'il peut changer de posture sans passer par une singularité. II y a donc plusieurs solutions dans un seul aspect. Autrement dit, cet aspect n'est plus un domaine d'unicité de l'opérateur géométrique $\mathbf{f}$.

Le nom "robot cuspidal" a été introduit en lien avec la condition d'existence d'un point singulier particulier de l'espace de travail appelé point cusp. On montre que si un point cusp existe, alors le robot est cuspidal [EW95]. La classification exhaustive des robots $3 R$ orthogonaux qui a été réalisée par la suite, montre que l'existence d'un point cusp est aussi une condition nécessaire pour ces robots [BWC04], [Bai04]. Beaucoup plus récemment en mars 2021, une preuve mathématique qu'il en est de même pour tous les robots $3 R$ venait d'être établie dans le cadre du projet ANR France-Autriche "ECARP" (https ://ecarp.lip6.fr/, projet en cours et preuve non encore publiée au moment de la rédaction de cet article). Cependant, on sait que cette condition nécessaire d'existence d'un point cusp ne tient plus pour les robots parallèles (voir $§ 5.7$ ). 

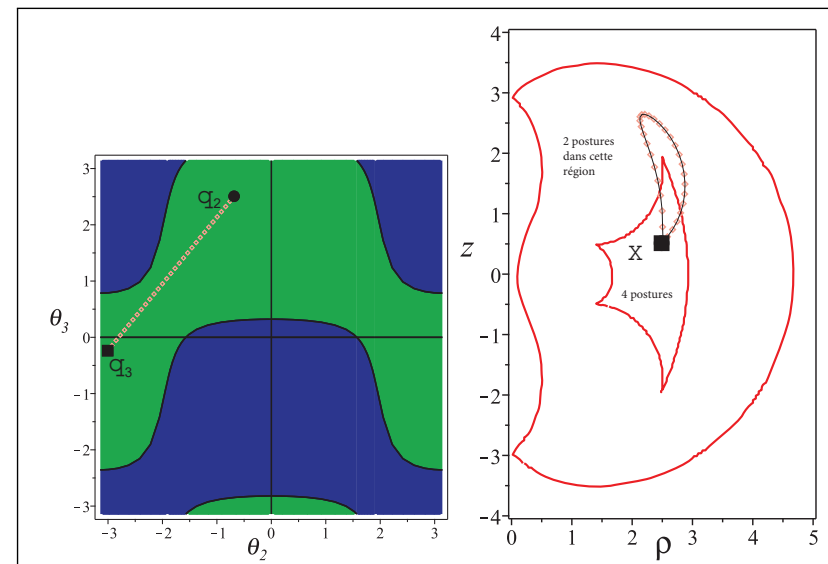

Figure 5 - Robot 3R cuspidal : les 2 aspects du domaine articulaire (à gauche, untés en radians) et section génératrice de l'espace de travail (à droite). On montre une trajectoire de changement de posture non singulier

Point cusp : Un point cusp (aussi appelé "fronce") est l'une des deux singularités stables formées lorsque l'on projette une surface pliée sur un plan, l'autre singularité étant un "pli" ("fold" en anglais) [Whi55]. Ces singularités sont dites stables car elles ne disparaissent pas sous l'effet d'une petite perturbation de la surface.

\section{Exemple 2 : Robot 3R cuspidal}

Reprenons le cas du robot $3 \mathrm{R}$ de l'exemple $1\left(d_{2}=\right.$ $1, d_{3}=2, d_{4}=1.5, r_{2}=1, r_{3}=0, \alpha_{2}=-90^{\circ}$ et $\alpha_{3}=90^{\circ}$ ).

On montre que le point $\mathbf{X}$ de coordonnées $x=2.5$, $y=0, z=0,5$ dans le repère $(O, x, y, z)$ (voir figure 5 à droite) est accessible selon quatre postures correspondant aux configurations articulaires suivantes (valeurs exprimées en radians) :

$$
\begin{array}{ll}
\mathbf{q 1}=[-1.8,-2.8,1.9]^{t}, & \mathbf{q} 2=[-0.9,-0.7,2.5]^{t} \\
\mathbf{q 3}=[-2.9,-3,-0.2]^{t}, & \mathbf{q 4}=[0.2,-0.3,-1.9]^{t}
\end{array}
$$

Les postures sont représentées en figure 6 .

D'après la figure 5 , on voit que les configurations q2 et q3 appartiennent au même aspect. Par conséquent, le passage de l'une à l'autre peut se réaliser sans franchissement de singularités, par exemple à l'aide d'une trajectoire linéaire (à gauche). La trajectoire reliant $\mathbf{q} \mathbf{2}$ et q3 génère alors une boucle dans l'espace de travail (à droite).

On montre que trois solutions coïncident lorsque le robot se trouve sur un point cusp. Cette propriété est très importante car elle permet de rechercher les points cusps comme les racines triples du polynôme caractéristique du modèle géométrique inverse.

Sous l'effet de l'opérateur géométrique $\mathbf{f}$, le domaine articulaire est "plié" le long des singularités du robot pour former plusieurs "couches", puis projeté dans l'espace de travail, formant des lignes de pli. Chaque couche correspond à une solution géométrique inverse du robot. La

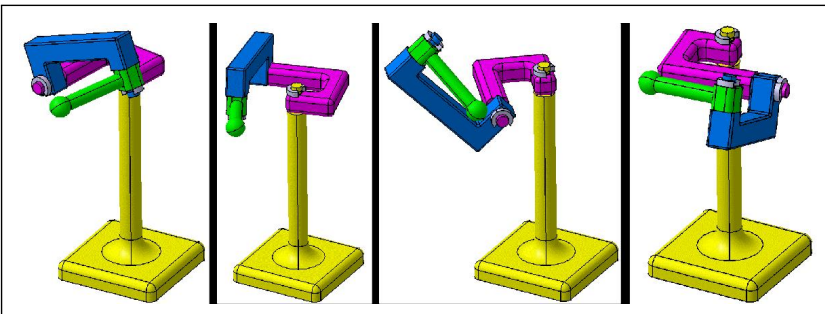

Figure 6 - Les 4 postures du robot 3R au point $x=2.5, y=0, z=0,5$.
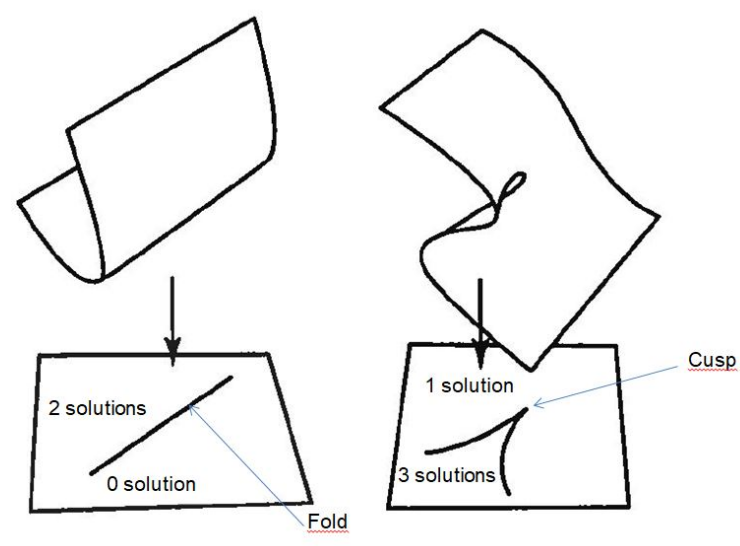

Figure 7 - Les deux singularités stables de type "fold" (à gauche) et "cusp" (à droite) obtenues en projetant une surface pliée sur un plan. A gauche, le pliage forme un simple pli ; à droite, il forme une fronce.

figure 7 illustre ce phénomène à l'aide d'une surface, représentant le domaine articulaire, se projetant sur un plan, représentant l'espace de travail. Avec un pliage en simple pli (à gauche), la zone à gauche de la ligne de pli dans le plan de projection (l'espace de travail) résulte de la projection de deux couches, correspondant à deux solutions inverses. Lorsque le pliage forme une fronce comme sur la figure 7 à droite, la projection donne deux lignes de pli qui se rejoignent en un point cusp, formant un point de rebroussement. Dans le plan de projection, la zone à l'intérieur des deux lignes de pli convergentes résulte de la projection de trois couches, correspondant à 3 solutions inverses.

\subsection{Changement de posture singulier et non singulier}

Un robot non cuspidal comme le robot anthorpomorphe de la figure 4 ne peut effectuer que des changements de posture singuliers. Lors d'un tel changement de posture, le robot effectue une trajectoire d'aller-retour vers une frontière de son espace de travail : en passant de la posture "coude haut" à la posture "coude bas", le robot anthropomorphe passe par la singularité "bras tendu". Ce faisant, l'organe terminal se déplace vers la frontière extérieure de son espace de travail, l'atteint puis repart vers sa pose initiale.

Pour un robot cuspidal, la figure 5, à droite, montre 


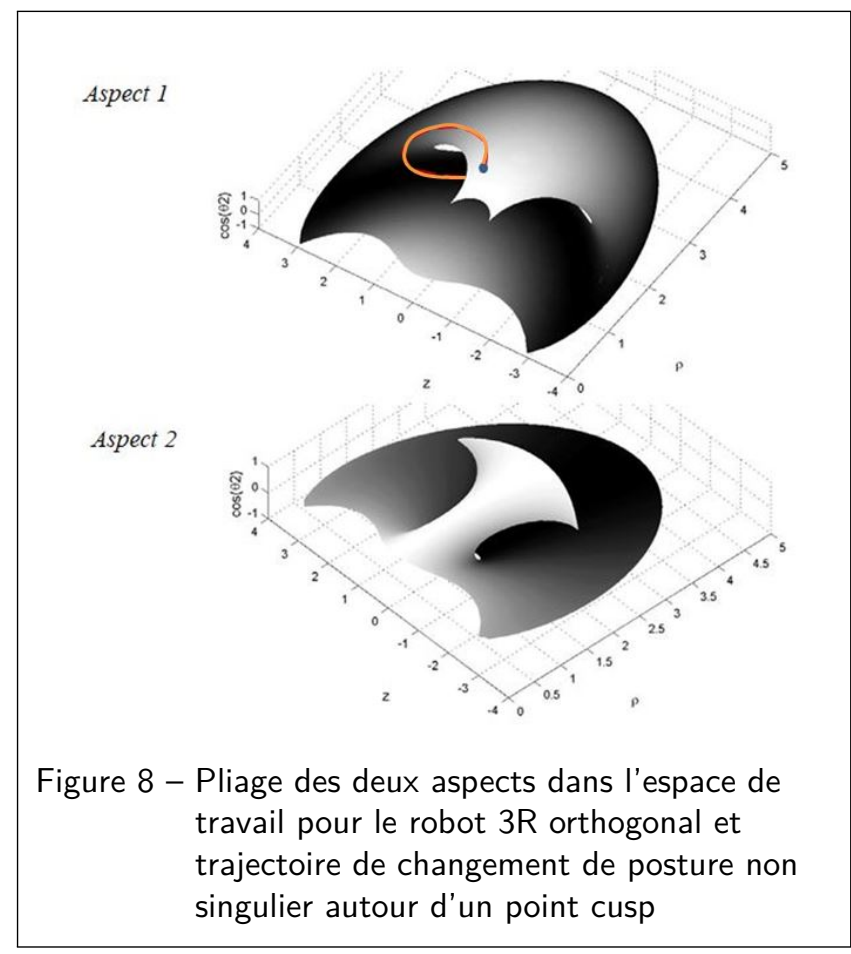

qu'un changement de posture non singulier s'effectue en tournant autour d'un point cusp [EW95]. Pour mieux comprendre ce phénomène, il est utile de montrer comment, pour ce robot 3R orthogonal, le domaine articulaire est "plié" avant d'être projeté sur l'espace de travail. Pour cela, on peut faire apparaître les "couches" associées aux différentes solutions géométriques inverses en traçant la section génératrice de l'espace de travail comme une surface dans l'espace $\left(\rho, z, \cos \left(\theta_{2}\right)\right.$. La coordonnée supplémentaire $\cos \left(\theta_{2}\right)$ permet de distinguer les différentes solutions inverses qui apparaissent alors sur des couches différentes. Le motif de pliage du domaine articulaire étant plus complexe que celui schématisé en figure 7, il est nécessaire de séparer le représentation selon les deux aspects (figure 8). II faut imaginer le schéma de pliage global en considérant que les deux surfaces correspondant aux deux aspects font en réalité partie d'une même surface pliée au niveau des bords extérieurs et d'une partie des bords intérieurs. La représentation séparée montre comment on peut passer d'une solution à l'autre dans un même aspect en contournant un point cusp.

Un robot cuspidal peut aussi effectuer un changement de posture singulier. C'est le cas lorsque le robot de la figure 5 doit changer de posture alors qu'il se situe dans la zone périphérique de son espace de travail. Le robot n'y admet que deux postures, une par aspect. Le changement de posture produira alors une trajectoire d'aller-retour vers la frontière extérieure de l'espace de travail, comme pour un robot non cuspidal. Lorsque le robot est dans la zone centrale, il y a quatre postures, deux dans chaque aspect. Si les postures initiale et finale sont dans deux aspects différents, le changement de posture sera singulier. Le robot effectuera alors une trajectoire d'aller-retour, soit vers la frontière intérieure qui sépare la région centrale de la région périphérique, soit vers la frontière extérieure. La figure 9 montre, à

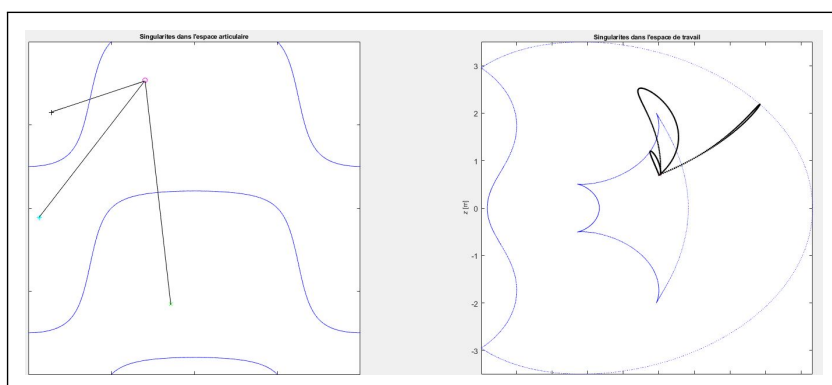

Figure 9 - Changements de posture singulier et non singulier d'un robot 3R cuspidal (dans l'espace articulaire à gauche, dans l'espace de travail à droite)

partir de la zone centrale, deux trajectoires de changement de posture singulier (trajectoires d'aller-retour vers la frontière intérieure à gauche et vers la frontière extérieure à droite) et une trajectoire de changement de posture non singulier (au centre, contournant le point cusp supérieur droit).

\subsection{Identification des robots cuspidaux}

On sait que s'il existe un point cusp dans l'espace de travail d'un robot, alors celui-ci est cuspidal. On sait aussi que lorsque le robot est de type $3 \mathrm{R}$ orthogonal, l'existence d'un point cusp est aussi une condition nécessaire. Autrement dit, s'il n'existe aucun point cusp dans l'espace de travail d'un robot 3R orthogonal, alors ce robot n'est pas cuspidal. Des méthodes numériques, graphiques ou algébriques peuvent être utilisées pour vérifier les conditions d'existence de points cusp. Elles constituent une aide intéressante pour le concepteur dans le choix d'un robot.

Étant donné que l'existence d'un point cusp prouve que le robot est cuspidal, ceci peut être utilisé comme critère de classification. On sait qu'un point cusp est associée à une racine triple du polynôme caractéristique du modèle géométrique inverse. On peut donc rechercher ces racines triples. S'il en existe au moins une, on peut affirmer que le robot est cuspidal. Les racines triples peuvent être calculées par le système suivant :

$$
\left\{\begin{array}{c}
P(t)=0 \\
\frac{\partial P(t)}{\partial t}=0 \\
\frac{\partial^{2} P(t)}{\partial t^{2}}=0
\end{array}\right.
$$

où $P(t)$ est le polynôme caractéristique. Le système (3) conduit à des équations de degré très élevé, difficiles à résoudre de façon symbolique avec les outils mathématiques courants [EI 96].

\section{Exemple 3 : Robot 3R orthogonal}

Considérons un robot $3 \mathrm{R}$ orthogonal tel que $r_{3}=$ 0 . En posant $d_{2}=1$ sans perte de généralité (normalisation), il ne reste que 3 paramètres à considérer : $d_{3}, d_{4}$ et $r_{2}$. Le modèle géométrique direct 
s'écrit alors :

$$
\left\{\begin{array}{c}
x=\left(d_{3}+d_{4} \cos \theta_{3}\right)\left(\cos \theta_{1} \cos \theta_{2}\right) \\
-\left(r_{2}+d_{4} \sin \theta_{3}\right) \sin \theta_{1}+\cos \theta_{1} \\
y=\left(d_{3}+d_{4} \cos \theta_{3}\right)\left(\sin \theta_{1} \cos \theta_{2}\right) \\
+\left(r_{2}+d_{4} \sin \theta_{3}\right) \cos \theta_{1}+\sin \theta_{1} \\
z=-\left(d_{3}+d_{4} \cos \theta_{3}\right) \sin \theta_{2}
\end{array}\right.
$$

Le modèle géométrique inverse s'obtient en éliminant deux des trois variables articulaires, par exemple $\theta_{1}$ et $\theta_{2}$. On obtient alors l'équation suivante [Pie68] :

$$
\begin{aligned}
& m_{5} \cos \theta_{3}{ }^{2}+m_{4} \sin \theta_{3}{ }^{2}+m_{3} \cos \theta_{3} \sin \theta_{3} \\
& +m_{2} \cos \theta_{3}+m_{1} \sin \theta_{3}+m_{0}=0
\end{aligned}
$$

où

$$
\begin{aligned}
& \left\{\begin{array}{c}
m_{0}=-x^{2}-y^{2}+r_{2}^{2}+\frac{(R+1-L)^{2}}{4} \\
m_{1}=2 r_{2} d_{4}+(L-R-1) d_{4} r_{2} \\
m_{2}=(L-R-1) d_{4} d_{3}
\end{array}\right. \\
& L=d 3^{2}+r 3^{2}+r 2^{2}+d 4^{2} \\
& R=\rho^{2}+z^{2} \\
& \text { et }\left\{\begin{array}{c}
m_{3}=2 r_{2} d_{3} d_{4}^{2} \\
m_{4}=d_{4}{ }^{2}\left(r_{2}{ }^{2}+1\right) \\
m_{5}=d_{3}{ }^{2} d_{4}^{2}
\end{array}\right.
\end{aligned}
$$

Le polynôme caractéristique s'obtient en remplaçant dans l'équation précédente les sinus et cosinus par la tangente de l'angle moitié $t=$ $\tan \left(\theta_{3} / 2\right)$. On obtient alors un polynôme de degré 4 en $t$, dont les coefficients dépendent de $R$, $z, d_{3}, d 4, r_{2}$ [Bai04]. Pour savoir si le robot est cuspidal ou non, ce polynôme doit admettre au moins une racine triple réelle. Ceci est équivalent à rechercher l'existence de solutions réelles au système suivant :

$$
\left\{\begin{array}{l}
P\left(t, d_{3}, d_{4}, r_{2}, R, z\right)=0 \\
\frac{\partial P}{\partial t}\left(t, d_{3}, d_{4}, r_{2}, R, z\right)=0 \\
\frac{\partial^{2} P}{\partial t^{2}}\left(t, d_{3}, d_{4}, r_{2}, R, z\right)=0 \\
\frac{\partial^{3} P}{\partial t^{3}}\left(t, d_{3}, d_{4}, r_{2}, R, z\right) \neq 0
\end{array}\right.
$$

où

- $t, R, z$ sont les variables;

- $d_{3}, d_{4}, r_{2}$ sont les paramètres.

Pour le robot de l'exemple $1\left(d_{2}=1, d_{3}=2\right.$, $d_{4}=1.5, r_{2}=1, r_{3}=0, \alpha_{2}=-90^{\circ}$ et $\alpha_{3}=90^{\circ}$ ), le système précédent admet 4 solutions réelles. Dans le plan $(\rho, z)$ correspondant à une section génératrice de l'espace de travail, ces valeurs sont :

$$
\begin{array}{rr}
C_{1}=[2.4655,-1.9987] & C_{2}=[1.3555,-0.5047] \\
C_{3}=[1.3555,0.5047] & C_{4}=[2.4655,1.99872]
\end{array}
$$

La figure 10 montre ces 4 points cusps entourés en vert.

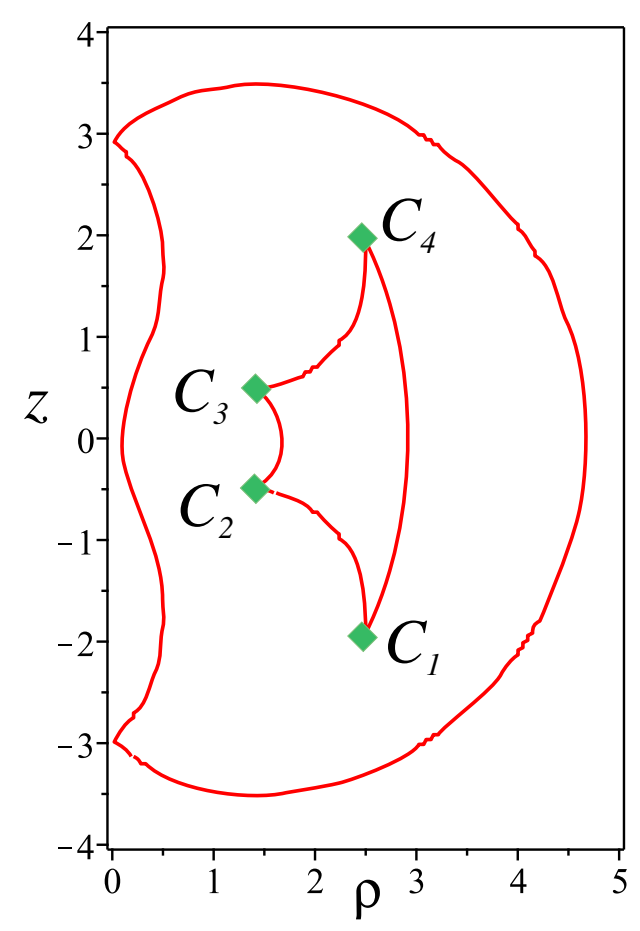

Figure 10 - Les 4 points cusp du robot $3 \mathrm{R}\left(d_{2}=1\right.$, $d_{3}=2, d_{4}=1.5, r_{2}=1, r_{3}=0$, $\alpha_{2}=-90^{\circ}$ et $\alpha_{3}=90^{\circ}$ )

A retenir : Si la plupart des robots sériels industriels doivent franchir une singularité au cours d'un changement de posture, cette propriété n'est pas vérifiée en général sur un robot quelconque. Un robot qui peut changer de posture sans passer par une singularité est dit cuspidal : il possède alors plusieurs solutions dans un même aspect. L'existence d'un point cusp dans son espace de travail est une condition suffisante pour que le robot soit cuspidal. Pour un robot $3 \mathrm{R}$ orthogonal, cette condition est également nécessaire. On peut ainsi identifier un robot cuspidal en recherchant l'existence de points cusp, ce qui se fait en résolvant un système d'équations algébriques. Lors d'un changement de posture non singulier, l'effecteur du robot suit une trajectoire fermée qui tourne autour d'un point cusp.

\section{Faisabilité de trajectoires}

\subsection{Régions t-parcourables pour un robot non cuspidal}

Région t-parcourable: Une région de l'espace de travail est dite t-parcourable si toute trajectoire continue dans cette région peut être parcourue par l'organe terminal sans jamais quitter la trajectoire en cours de déplacement. Pour un robot non-cuspidal, les régions tparcourables sont les régions accessibles dans un même aspect [WC91].

Pour un robot non cuspidal, il a été démontré que les aspects définissent les régions t-parcourables [BL86], [WC91]. Cette propriété est très intéressante pour l'uti- 


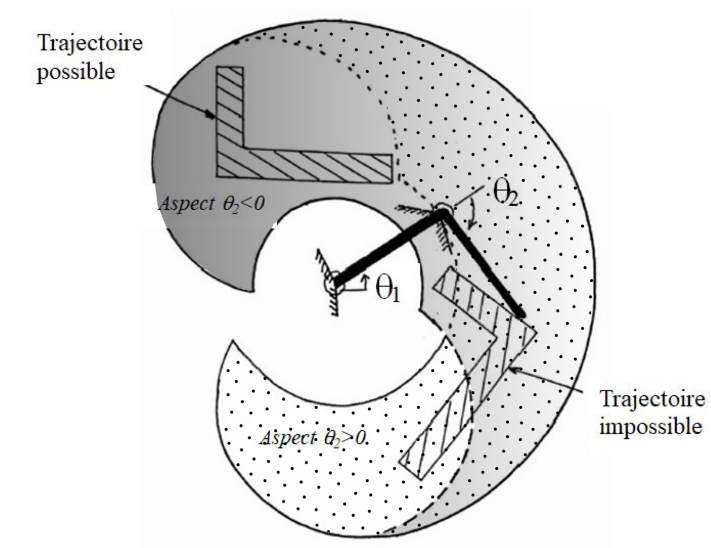

Figure 11 - Utilisation des régions t-parcourables pour le placement de pièces

lisateur ou le concepteur d'un site robotisé car elle lui donne une information globale sur les performances du robot dans son espace de travail. En l'absence d'obstacles susceptibles de gêner les mouvements du robot, on peut obtenir les régions t-parcourables à partir de leurs frontières. On trace l'image des singularités et des limites articulaires dans l'espace de travail à l'aide de l'opérateur géométrique direct $\mathbf{f}$.

\section{Exemple 4 : Utilisation des régions $t$ - parcourables}

La figure 11 montre les deux régions tparcourables d'un robot SCARA qui correspondent aux aspects $\theta_{2}<0$ (motif gris) et $\theta_{2}>0$ (motif pointillé). Le robot possède des limites articulaires, matérialisées par deux demi-segments hachurés sur chaque articulation. Les deux régions t-parcourable se chevauchent (motif gris et pointillé), leur frontière extérieure commune étant un arc de cercle dont le rayon est la somme des deux longueurs de bras et qui correspond à la singularité "bras tendu" définie par $\theta_{2}=0$. Les autres frontières des régions sont des arcs de cercles de rayon plus petits définis par les limites articulaires. Les frontières montrées en traits discontinus délimitent la zone de chevauchement. Pour une tâche de découpe de pièces en forme de "L", le concepteur placera la pièce de telle sorte que la totalité du contour se trouve intégralement dans une région t-parcourable, ce qui est le cas pour le placement du haut, mais pas du bas puisque les extrémités du contour se trouvent alors dans deux régions tparcourables différentes.

\subsection{Régions t-parcourables pour un robot cuspidal}

L'existence de robots cuspidaux a des conséquences majeures sur l'étude de faisabilité de trajectoires. En effet, lorsqu'il existe plus d'une solution dans un aspect, celuici ne garantit plus l'existence de trajectoires continues.

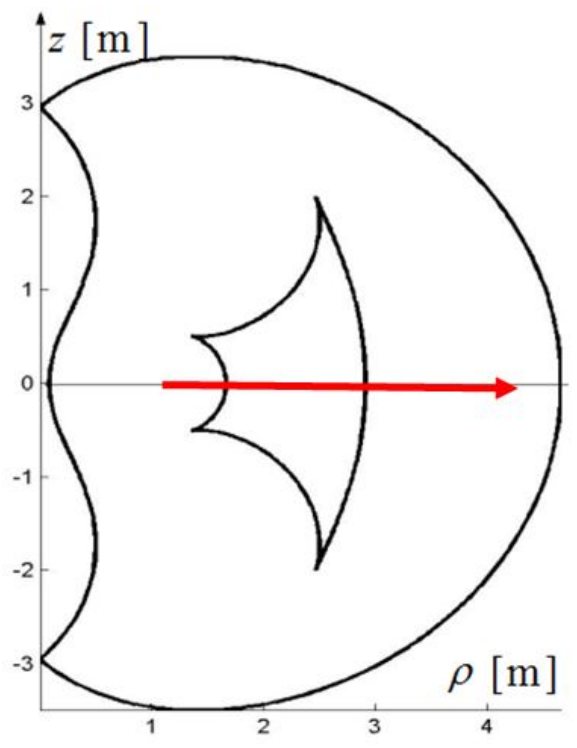

Figure 12 - Trajectoire dans un seul aspect qui n'est pas faisable

La figure 12 montre une trajectoire totalement accessible dans un même aspect du robot $3 R$ orthogonal mais qui n'est pourtant pas réalisable. Pour s'en convaincre, on peut se reporter à la figure 8 : on voit que quelque soit l'aspect choisi, le robot sera stoppé sur un bord intérieur de l'aspect. Plus généralement, on voit qu'il n'est pas possible de traverser successivement les deux frontières intérieures gauche et droite qui délimitent la zone à 4 solutions.

Pour un robot cuspidal, les régions t-parcourables ne peuvent donc plus être définies à partir des aspects. Il est nécessaire de définir des nouveaux domaines d'unicité.

Pour cela, il faut introduire de nouvelles frontières qui auront pour but de séparer les multiples solutions au sein d'un aspect. Ces frontières sont appelées surfaces caractéristiques et sont définies pour chaque aspect [Wen04].

Les surfaces caractéristiques : Les surfaces caractéristiques d'un aspect $\mathcal{A}_{j}$, notées $\mathcal{S}_{C}\left(\mathcal{A}_{j}\right)$, sont définies comme l'image réciproque dans $\mathcal{A}_{j}$ de l'image $\mathbf{f}\left(\mathcal{A}_{\mathbf{j}}^{*}\right)$ des frontières $\mathcal{A}_{j}^{*}$ qui délimitent $\mathcal{A}_{j}$ :

$$
\mathcal{S}_{C}\left(\mathcal{A}_{j}\right)=\mathbf{f}^{-\mathbf{1}}\left(\mathbf{f}\left(\mathcal{A}_{\mathrm{j}}^{*}\right)\right) \cap \mathcal{A}_{\mathbf{j}}
$$

Avec :

- f : l'opérateur géométrique,

- $\mathcal{A}_{j}^{*}$ : les frontières de l'aspect $\mathcal{A}_{j}$,

$-\mathbf{f}^{-1}\left(\mathbf{f}\left(\mathcal{A}_{\mathbf{j}}^{*}\right)\right)=\left\{\mathbf{q} / \mathbf{f}(\mathbf{q}) \in \mathbf{f}\left(\mathcal{A}_{\mathbf{j}}^{*}\right)\right\}$,

Pour mieux comprendre cette définition, prenons le cas du robot $3 \mathrm{R}$ orthogonal. La figure 8 nous montre que, dans un même aspect, chaque point d'une frontière intérieure de l'espace de travail est accessible par deux solutions géométriques inverses. L'une est une configuration singulière et correspond à un bord, par exemple pour une position située sur l'un des bords de la "couche" supérieure de l'aspect. L'autre est non singulière 


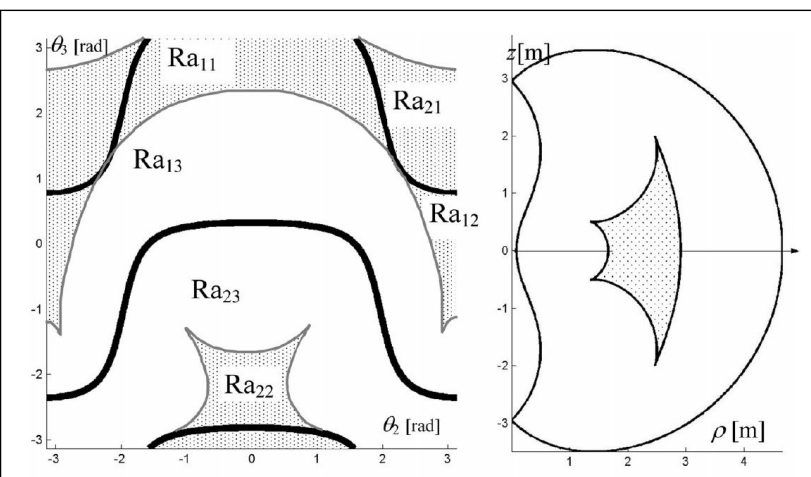

Figure 13 - A gauche : singularités (en traits gras) et surfaces caractéristiques (en traits fins) pour le robot $3 \mathrm{R}$ orthogonal $\left(d_{2}=1\right.$, $\left.d_{3}=2, d_{4}=1.5, r_{2}=1, r_{3}=0\right)$. Les régions grisées correspondent à la région centrale grisée de l'espace de travail (à droite)

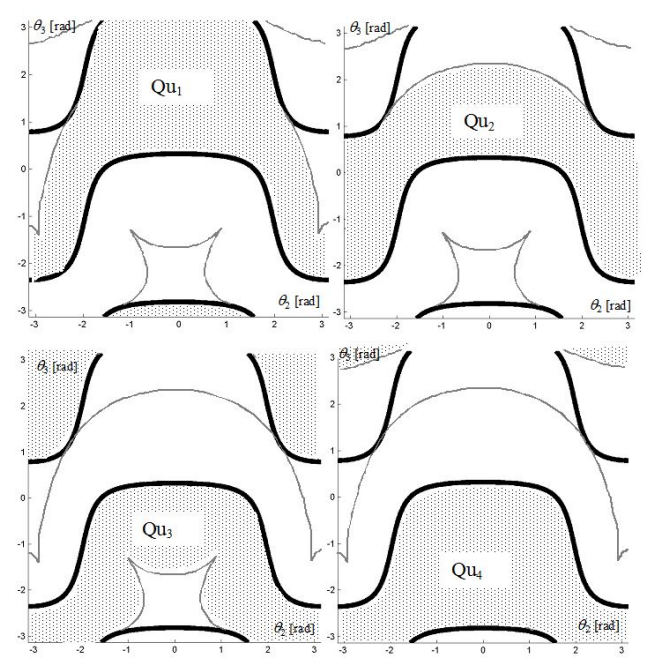

Figure 14 - Les 4 domaines d'unicité pour le robot 3R orthogonal $\left(d_{2}=1, d_{3}=2, d_{4}=1.5\right.$, $\left.r_{2}=1, r_{3}=0\right)$

et correspond à la même position mais située sur la "couche" inférieure. Les surfaces caractéristiques correspondent donc aux configurations articulaires non singulières d'un aspect associées à des points situés sur les frontières intérieures de l'espace de travail.

La figure 13 à gauche montre les surfaces caractéristiques du robot $3 \mathrm{R}$ orthogonal. Rappelons que même si la figure montre des courbes, il s'agit bien de surfaces, tout comme les singularités, car l'espace articulaire est en réalité de dimension 3. En se reportant à la figure 5 à gauche, on voit que les surfaces caractéristiques séparent bien les deux solutions inverses dans l'aspect.

Remarque: Pour un robot non cuspidal, la formule 9 donne l'ensemble vide. Autrement dit, un robot non cuspidal n'a pas de surfaces caractéristiques, ce qui est logique puisqu'il n'a qu'une seule solution par aspect.

Les surfaces caractéristiques permettent de définir de nouveaux domaines d'unicité. En effet, on peut montrer que les surfaces caractéristiques réalisent une partition des aspects en domaines où le robot n'admet qu'une seule solution inverse [Wen04]. Sur la figure 13, ces domaines sont identifiés par $R a_{i j}$. La figure montre en grisé les domaines qui correspondent à la région centrale de l'espace de travail. Comme il y a quatre solutions dans cette région centrale, il y a quatre domaines associées dans l'espace articulaire. Néanmoins, ces domaines ne sont pas les domaines d'unicité les plus grands. Pour obtenir ces derniers, il faut prendre la réunion de plusieurs domaines adjacents, comme expliqué dans [Wen04], où une méthode numérique est proposée pour les calculer. La figure 14 montre les 4 domaines d'unicité $Q u_{i}$ obtenus pour le robot $3 \mathrm{R}$ orthogonal : le domaine d'unicité $Q u_{1}$, par exemple, est obtenu comme la réunion des domaines $R a_{13}$ et $R a_{11}$ de la figure 13 .

Les régions parcourables sont alors obtenues comme les images des domaines d'unicité les plus grands dans l'espace de travail. La figure 15 montre les 4 régions $t$ parcourables $W f_{i}$ du robot $3 \mathrm{R}$ orthogonal. Les régions $W f_{1}$ et $W f_{2}$ sont associées à l'aspect 1 , tandis que les régions $W f_{3}$ et $W f_{4}$ sont associées à l'aspect 2. Les lignes intérieures constituent des coupures correspondant à des lignes de points inaccessibles. II n'est donc pas possible de les franchir. On vérifie bien que la trajectoire horizontale de la figure 12 n'est pas faisable puisqu'elle rencontre des lignes infranchissables dans chacune des régions t-parcourables. En revanche, le robot pourrait suivre la trajectoire si celle-ci était verticale. II suffirait d'utiliser la région $W f_{2}$ si le robot se trouve initialement dans l'aspect 1 , ou $W f_{4}$ s'il est dans l'aspect 2 . Le choix de l'une ou l'autre région dépendra de la solution inverse choisie pour suivre la trajectoire.

\subsection{Influence des limites articulaires}

Les surfaces caractéristiques et les régions tparcourables des robots cuspidaux ont été illustrées avec un robot $3 \mathrm{R}$ orthogonal sans limites articulaires, mais la théorie et les définitions s'appliquent aussi lorsque les débattements articulaires sont limités. En pratique, les limites articulaires vont supprimer des singularités dans le domaine articulaire (celles situées au delà des limites articulaires). En même temps, elles vont produire de nouvelles frontières pour les aspects, définies par les limites articulaires. Dans l'espace de travail, une partie des frontières vont disparaître, tandis que de nouvelles vont apparaître. En cas de limites articulaires serrées, il est possible que tous les points cusps disparaissent : un robot cuspidal peut alors devenir non cuspidal. Le lecteur intéressé pourra trouver des exemples dans [El 96] et [Wen04]. 


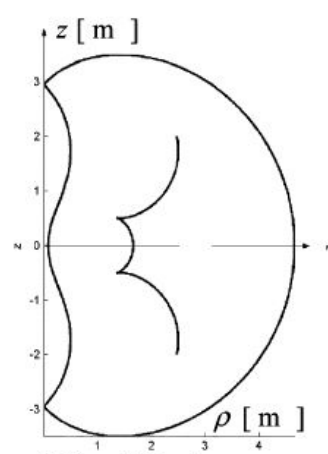

$\mathrm{Wf}_{1}=\mathrm{f}\left(\mathrm{Qu}_{1}\right)$

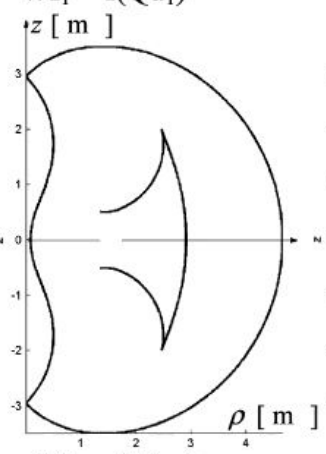

$\mathrm{Wf}_{3}=\mathrm{f}\left(\mathrm{Qu}_{3}\right)$

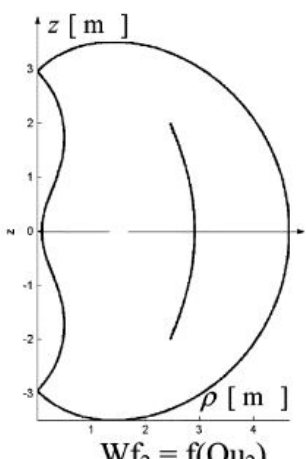

$\mathrm{Wf}_{2}=\mathrm{f}\left(\mathrm{Qu}_{2}\right)$

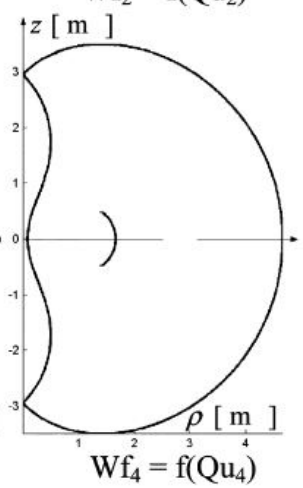

Figure 15 - Les 4 régions t-parcourables pour le robot 3R orthogonal $\left(d_{2}=1, d_{3}=2, d_{4}=1.5\right.$, $\left.r_{2}=1, r_{3}=0\right)$

A retenir : L'étude de faisabilité de trajectoires continues est très importante pour des tâches de process par exemple. Or, cette étude diffère selon que le robot est cuspidal ou non. Pour un robot cuspidal, les régions tparcourables de l'espace de travail, c'est-à-dire les régions où toute trajectoire continue est faisable, sont les images des aspects. Pour un robot non cuspidal, les régions t-parcourables sont plus difficiles à calculer et nécessite la détermination de nouveaux domaines d'unicité dans le domaine articulaire. Pour cela, de nouvelles surfaces qui séparent les solutions inverses, appelées surfaces caractéristiques, doivent être définies dans le domaine articulaire. On obtient alors les régions tparcourables comme les images des domaines d'unicités obtenus à partir de ces surfaces caractéristiques.

\section{Enumération et classification des robots cuspidaux et non- cuspidaux}

\subsection{Conditions géométriques simples conduisant à des robots non- cuspidaux}

Plusieurs conditions géométriques simples conduisant à des robots $3 R$ non cuspidaux ont pu être établies. Certaines conditions correspondent aux robots "résolubles" au sens de Pieper [Pie68; KD99], c'est-à-dire dont le modèle géométrique inverse peut se résoudre à l'aide de polynômes de degré au maximum égal à 2 . De tels ro- bots ne peuvent pas avoir de point cusp puisqu'aucune racine triple ne peut exister avec des polynômes de degré 2 , il sont donc non cuspidaux. Ainsi, les six conditions suivantes ont été établies [Wen97] :

1. les deux premiers axes sont parallèles;

2. les deux derniers axes sont parallèles;

3. les deux premiers axes se coupent;

4. les deux derniers axes se coupent;

5. les deux premiers axes sont orthogonaux et $r_{2}=$ $r_{3}=0$;

6. les axes sont orthogonaux deux à deux et $r_{2}=0$;

Les quatre premières conditions correspondent aux robots résolubles de Pieper et sont usuelles sur les robots industriels. En revanche, les deux dernières conditions sont moins courantes.

Il est intéressant de noter que lorsque les axes sont orthogonaux deux à deux (robot orthogonal), le robot est cuspidal lorsque $r_{2} \neq 0$ et $r_{3}=0$ mais ne l'est plus lorsque $r_{2}=0$ et $r_{3} \neq 0$.

Par ailleurs, on peut montrer que lorsque deux de ses articulations sont prismatiques, un robot sériel à trois degrés de liberté est nécessairement non cuspidal [EI 96]. Avec une seule articulation prismatique, des conditions géométriques équivalentes aux conditions 1 à 4 ci-dessus existent également [EI 96].

\subsection{Classification des robots $3 \mathrm{R}$ orthogo- naux}

On a vu que l'existence d'un point cusp, correspondant à un point triple du polynôme caractéristique, indique que le robot est cuspidal. En recherchant les robots dont le polynôme caractéristique a au moins un point triple, on obtiendra des robots cuspidaux.

Pour cela, partant du système (3), on peut rechercher les conditions sur les paramètres géométriques pour que ce système admette des solutions réelles. La tâche est assez ardue et d'une grande complexité algébrique en général. Cependant, il est possible de résoudre ce problème pour la famille des robots $3 \mathrm{R}$ orthogonaux à partir du système (6). Il faut pour cela avoir recours à des outils algébriques sophistiqués. Ces outils, qui s'appuient entre autres sur les bases de Groebner et la décomposition cylindrique algébrique [CR04], sont aujourd'hui disponibles dans le logiciel de calcul formel Maple, au travers de la librairie Siropa [Cha+19]. Afin de limiter le nombre de paramètres à 3 et simplifier les calculs, on commence par étudier une sous-famille de robots pour lesquels $r_{3}=0$, puis on normalise le problème en posant, sans perte de généralité, $d_{2}=1$. En recherchant les conditions pour lesquelles le nombre de solutions au système (6) change, on obtient 4 surfaces discriminantes $C_{1}, C_{2}, C_{3}, C_{4}$ dans l'espace des paramètres $\left(d_{2}, d_{3}, r_{2}\right)$. Ces surfaces sont définies par les équations suivantes:

$C_{1}: \quad d_{4}=\sqrt{\frac{1}{2}\left(d_{3}{ }^{2}+r_{2}{ }^{2}-\frac{\left(d_{3}{ }^{2}+r_{2}{ }^{2}\right)^{2}-\left(d_{3}{ }^{2}-r_{2}{ }^{2}\right)}{A B}\right)}$ 


$$
\begin{array}{ll}
C_{2}: & d_{4}=\frac{d_{3}}{1-d_{3}} B \text { et } \quad d_{3}<1 \\
C_{3}: & d_{4}=\frac{d_{3}}{d_{3}-1} B \text { et } \quad d_{3}>1 \\
C_{4}: & d_{4}=\frac{d_{3}}{1-d_{3}} B \text { et } \quad d_{3}<1
\end{array}
$$

où :

$$
A=\sqrt{\left(d_{3}+1\right)^{2}+r_{2}{ }^{2}} \text { et } B=\sqrt{\left(d_{3}-1\right)^{2}+r_{2}{ }^{2}}
$$

Ces surfaces réalisent une partition de l'espace des paramètres en domaines correspondant à des robots dont le nombre de points cusps est constant. Pour connaître le nombre de cusps dans chaque domaine, il suffit d'y choisir un robot quelconque et de compter ses points cusps. La figure 16 montre une section de l'espace des paramètres en $r_{2}=1$. On montre que les robots des domaines bleus ont 4 cusps, ceux du domaine blanc en ont 2 et ceux des domaines gris n'ont aucun cusps, ce sont donc des robots non cuspidaux. La figure 17 montre l'espace de travail pour un robot dans chaque domaine. Le nombre de solutions dans chaque région de l'espace de travail est indiqué. On voit bien que les robots issus des domaines 1 et 5 , correspondant aux robots non cuspidaux, ont une frontière intérieure sans cusps. Dans le domaine 1 , la frontière intérieure délimite un "trou" dans l'espace de travail, tandis que dans le domaine 5 , la frontière intérieure délimite une région à 4 solutions inverses. Dans les deux cas, le nombre de solutions autour de la région centrale est de 2 . En fait, le domaine 1 regroupe l'ensemble des robots n'ayant que deux solutions au maximum, alors que dans tous les autres domaines, les robots ont jusqu'à 4 solutions.

\subsection{Condition nécessaire et suffisante de cuspidalité d'un robot $3 \mathrm{R}$ orthogonal}

La classification de l'espace des paramètres permet d'identifier tous les robots cuspidaux et non cuspidaux de la famille étudiée. En utilisant les équations des surfaces discriminantes délimitant les domaines 1 et 5 et en relâchant la normalisation $d_{2}=1$, on peut écrire qu'un robot $3 \mathrm{R}$ orthogonal tel que $r_{3}=0$ est non cuspidal si et seulement si :

$$
\left\{\begin{array}{l}
d_{4}<\sqrt{\frac{1}{2}\left(d_{3}^{2}+r_{2}^{2}-\frac{\left(d_{3}^{2}+r_{2}^{2}\right)^{2}-d_{2}^{2}\left(d_{3}^{2}-r_{2}^{2}\right)}{\sqrt{\left(d_{3}+d_{2}\right)^{2}+r_{2}^{2}} \sqrt{\left(d_{3}-d_{2}\right)^{2}+r_{2}^{2}}}\right)} \\
\text { ou } \\
d_{3}<d_{2} \text { et } d_{4}>\frac{d_{3}}{d_{2}-d_{3}} \sqrt{\left(d_{3}-d_{2}\right)^{2}+r_{2}^{2}}
\end{array}\right.
$$

Cette condition est très utile pour le concepteur désirant mettre en oeuvre un robot orthogonal qui ne soit pas cuspidal. Notons que les robots non cuspidaux du domaine 5, c'est-à-dire satisfaisant la seconde condition de (15), sont plus intéressants car ils possèdent une région à 4 solutions dans leur espace de travail, tandis que ceux du domaine 1 ont un trou et aucune région à 4 solutions.

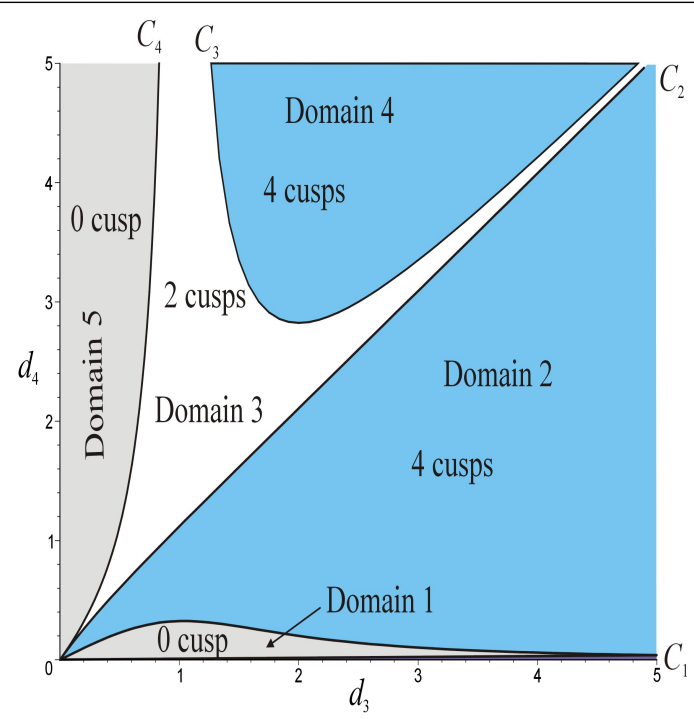

Figure 16 - Partition de l'espace de paramètres en fonction du nombre de points cusps pour les robots $3 \mathrm{R}$ orthogonaux normalisés par $d_{2}=1$ et tels que $r_{3}=0$ (section en $r_{2}=1$ )

Lorsque $r_{3} \neq 0$, l'espace des paramètres est de dimension 4 . En procédant de la même façon que précédemment, on obtient des hyper-surfaces discriminantes d'équation très complexe [Bai04]. L'une d'elles est de degré 12 et contient pas moins de 536 termes! De plus, alors que les robots n'ont que 4 points cusps au maximum lorsque $r_{3}=0$, il peut exister jusqu'à 8 points cusps lorsque $r_{3} \neq 0$. La figure 18 montre une section de l'espace des paramètres en $r_{2}=0.3, r_{3}=0.8$. Le nombre de points cusps est indiqué dans chaque domaine.

\subsection{Cas des robots à 6 articulations}

\subsubsection{Robots à poignet rotule}

La plupart des robots industriels sont conçus en ajoutant un poignet rotule au bout d'un robot 3 axes. Un poignet rotule (parfois appelé aussi "sphérique") est réalisé par la mise en série de trois articulations pivots à axes concourants et généralement orthogonaux deux à deux, ce qui permet de réaliser une rotule actionnée. La figure 19 montre un robot anthropomorphe analogue à celui de la figure 2 , constitué d'un porteur $3 R$ avec un poignet rotule $3 \mathrm{R}$. Cette stratégie de conception permet de simplifier les modèles géométriques du robot ainsi que ses singularités en découplant les équations de positions de celles d'orientation [KD99]. Ce découplage permet aussi de découpler l'analyse de cuspidalité du robot. Sachant que le poignet rotule est un mécanisme simple qui est non cuspidal (tous ses axes se coupent, il entre donc dans les conditions de non-cuspidalité énoncées au paragraphe 4.1), un robot à 6 articulations et poignet rotule sera cuspidal si et seulement le robot constitué par les 3 premiers axes, appelé porteur, est cuspidal. Tous les résultats de classification et d'analyse présentés précédemment restent donc valables pour les robots 


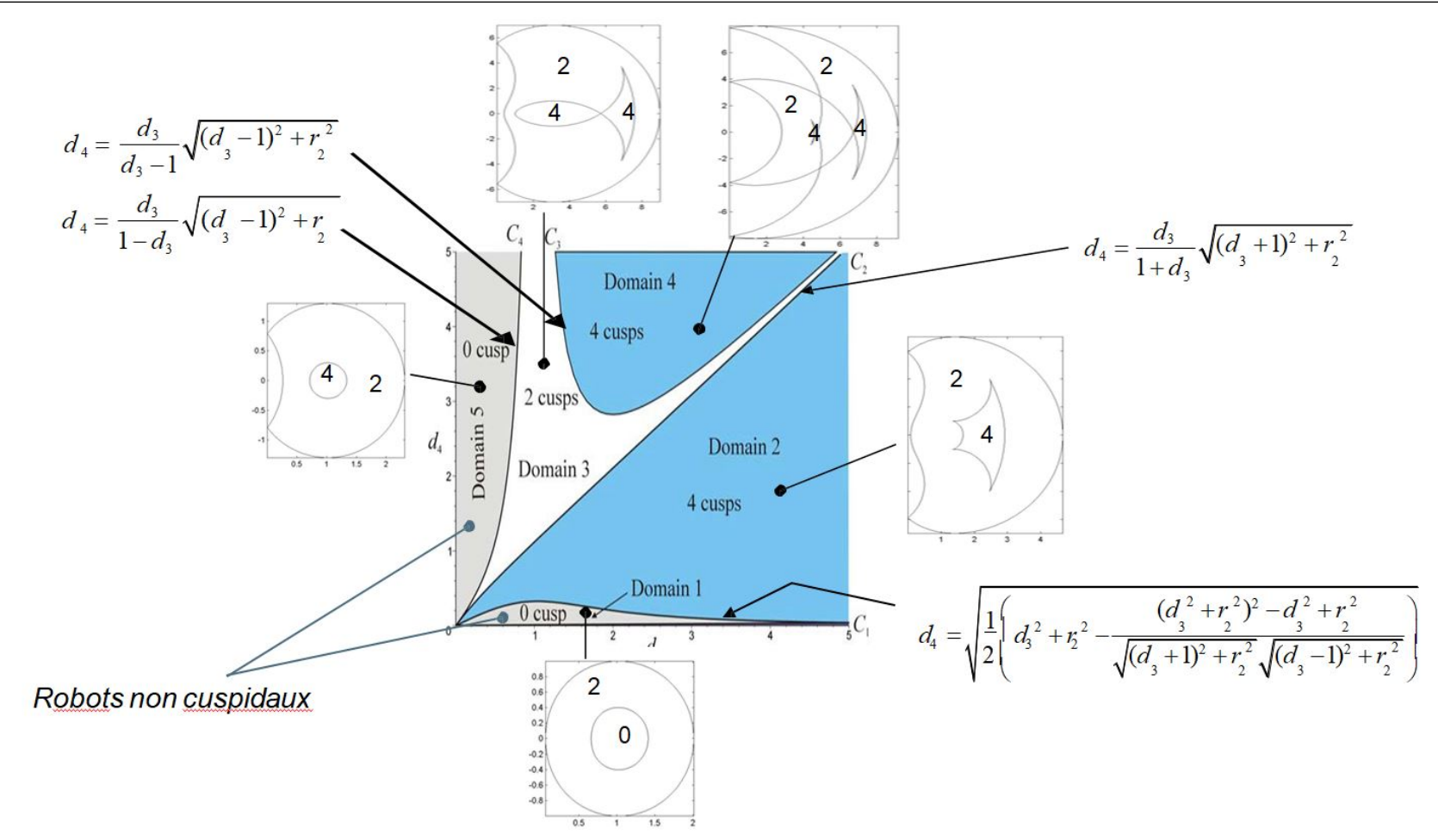

Figure 17 - Exemples d'espaces de travail dans chaque domaine de l'espace des paramètres pour des robots normalisés par $d_{2}=1$ (section en $r_{2}=1$ ). On a reporté l'équation de chaque surface discriminante. Le nombre de solutions accessibles dans chaque région de l'espace de travail est indiqué.

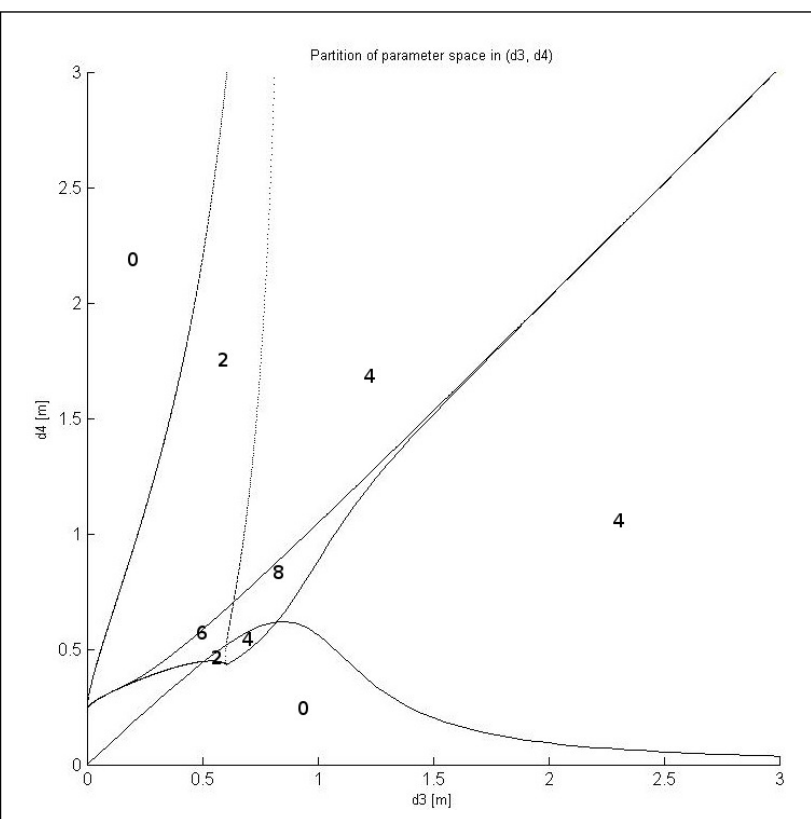

Figure 18 - Partition de l'espace de paramètres en fonction du nombre de points cusps pour les robots $3 \mathrm{R}$ orthogonaux lorsque $r_{3} \neq 0$ (section en $r_{2}=0.3, r_{3}=0.8$ )

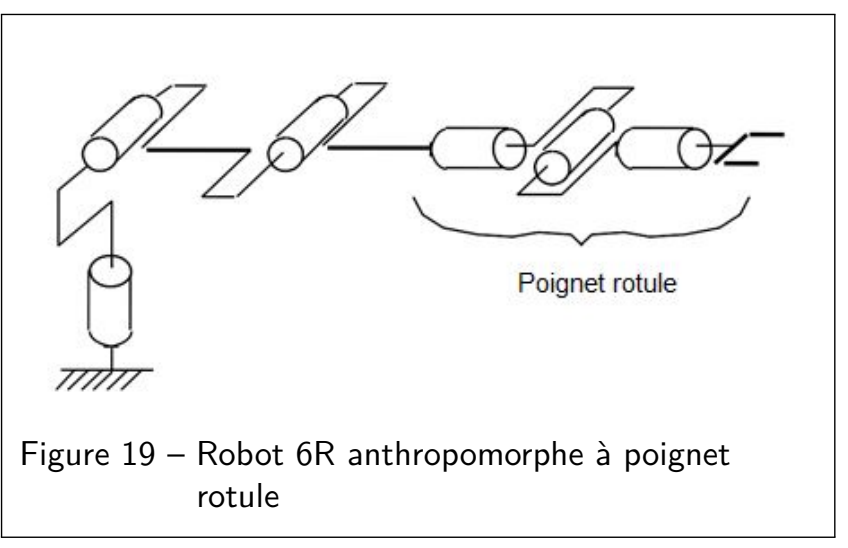

à 6 articulations et poignet rotule.

A notre connaissance et à la date de rédaction de cet article, il n'existe pas d'exemple de robot industriel à poignet rotule qui soit cuspidal. Un grand constructeur de robots, ABB, avait pourtant commercialisé en 1998 un robot à poignet rotule qui s'est avéré cuspidal. II s'agissait du robot IRB 6400C (Fig. 20. Sa particularité résidait dans la permutation des deux premiers axes par rapport à un robot anthropomorphe, rendant tous ses axes mutuellement orthogonaux : on avait donc affaire à un porteur orthogonal tel que ceux étudiés dans cet article. Le but recherché par le concepteur était de limiter le volume balayé afin de pouvoir disposer les robots plus près les uns des autres le long des lignes d'assemblage de véhicules. Au moment de sa sortie, les résultats sur la classification des robots orthogonaux n'étaient pas encore publiés. Ce robot a été supprimé du catalogue car 
les utilisateurs rencontraient des difficultés pour planifier des trajectoires. En fait, ce robot s'est avéré être cuspidal. Le concepteur, s'il avait eu connaissance de la classification, aurait pu facilement régler les paramètres géométriques pour que le porteur de ce robot tombe dans le domaine 5 des robots $3 \mathrm{R}$ orthogonaux non cuspidaux à 4 solutions [Wen07].

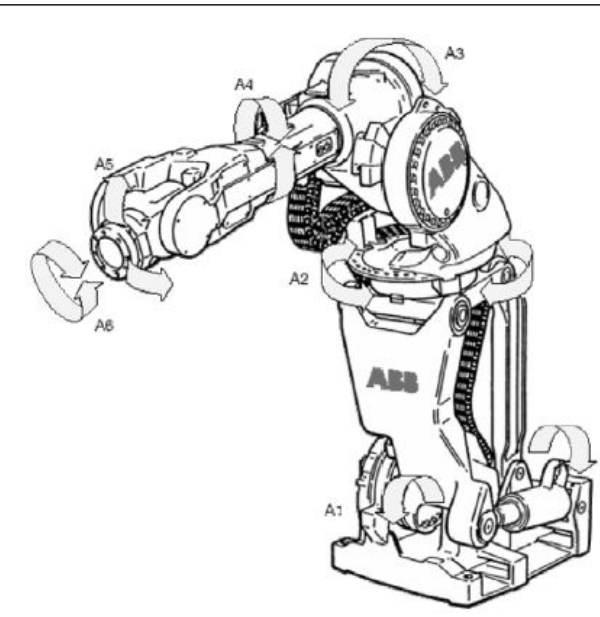

Figure 20 - Robot industriel cuspidal à poignet rotule et à porteur orthogonal (ABB IRB 6400C).

\subsubsection{Robots sans poignet rotule}

II existe très peu de résultats sur les robots sans poignet rotule, car ils sont bien plus difficiles à analyser. En effet, l'absence de découplage entre positions et orientations rend la résolution du modèle géométrique inverse complexe (voir par exemple [Pfu09] pour la proposition d'un algorithme qui s'appuie sur le formalisme de Study). De plus, le déterminant de la matrice jacobienne ne se factorise généralement pas et dépend de plus de 3 variables articulaires, ce qui rend difficile l'identification et le comptage des aspects. II existe toutefois quelques exemples de robots sans poignet rotule qu'il a été possible d'analyser :

- Les robots $6 \mathrm{R}$ à trois axes parallèles : cette particularité permet un découplage analogue aux robots à poignet rotule [KD99]. Comme les robots anthropomorphes, ces robots sont non-cuspidaux, ont 8 aspects et admettent jusqu'à 8 postures. On peut citer par exemple les robots industriels UR5 et UR10 de la société "Universal Robots" [CDS20] (figure 21, en haut) ou, bien plus ancien, le robot Schilling Titan II à actionnement hydraulique et généralement utilisé comme bras manipulateur téléopéré [Deb+01] (figure 21, en bas);

- Les robots anthropomorphes avec offset au poignet : il s'agit de robots $6 \mathrm{R}$ dérivés de la famille des robots anthropomorphes, pour lesquels un décalage a été introduit le long de l'axe 5, c'est-à-dire du deuxième axe du poignet. Ce décalage fait que les trois axes du poignet ne se coupent plus. II a pour but de supprimer la singularité de poignet, particulièrement gênante pour des applications de process.
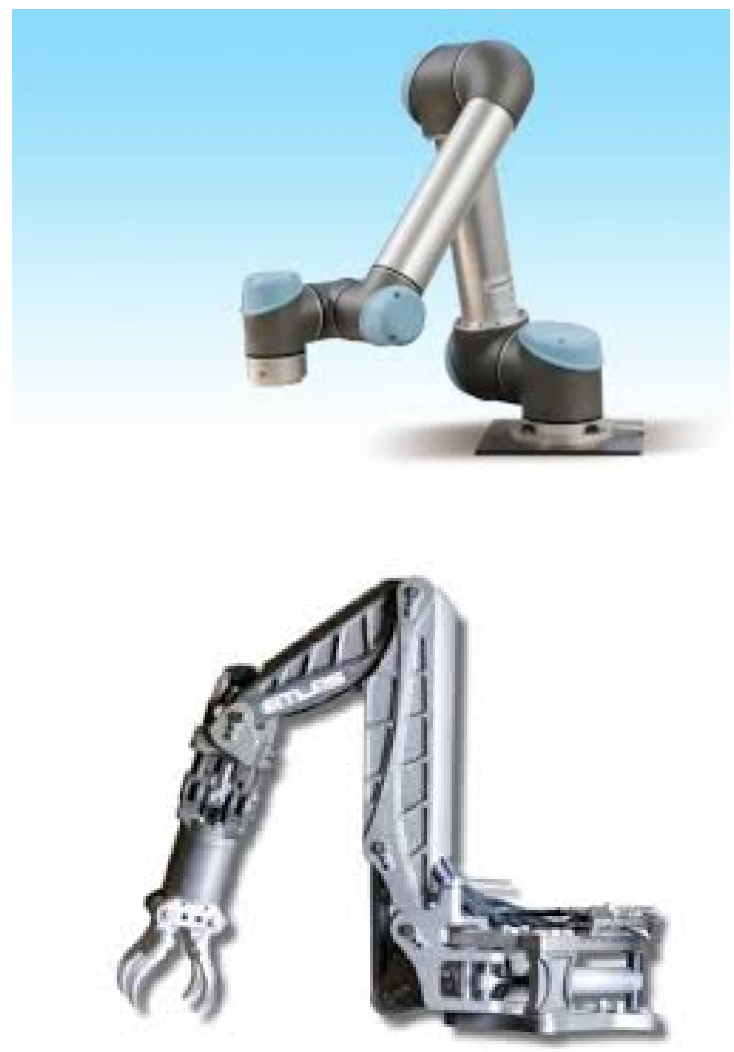

Figure 21 - Deux robots industriels $6 \mathrm{R}$ avec 3 axes parallèles : le robot UR10 de la société Universal Robots (en haut) et le robot Schilling Titan II (en bas)

Plusieurs robots industriels de peinture possèdent cette caractéristique, comme par exemple le robot GMF P150 (figure 22, en haut) ou, plus récent, le robot Fanuc P250iB (figure 22, en bas).

Sur un modèle numérique du GMF P150, on a pu mettre en évidence une trajectoire de changement de posture non singulier, montrant ainsi son caractère théorique cuspidal. Néanmoins, la présence de limites articulaires fortes réduit à 2 le nombre de postures de ce robot, restant alors toujours séparées par une singularité [EI 96]. Le robot est donc, en pratique, non cuspidal. Le robot Fanuc P250iB, plus récent, n'a pas encore été analysé mais, d'architecture analogue au robot GMF P150, il serait vraisemblablement aussi cuspidal sans ses limites articulaires.

- Les robots d'Innocenti : Deux robots 6R cuspidaux imaginaires ont été analysés dans [PI88] pour mettre en évidence l'existence de robots cuspidaux (voir historique en début de chapitre). L'un de ces robots a une géométrie très simple : il y a trois paires d'axes orthogonaux: $(1,2),(3,4),(5,6)$ et deux paires d'axes parallèles : $(2,3)$ et $(4,5)$ (voir figure 23 à gauche). L'autre robot a une architecture quelconque (23 à droite). Pour chacun de ces robots, les auteurs ont trouvé numériquement une trajectoire non singulière permettant de passer 

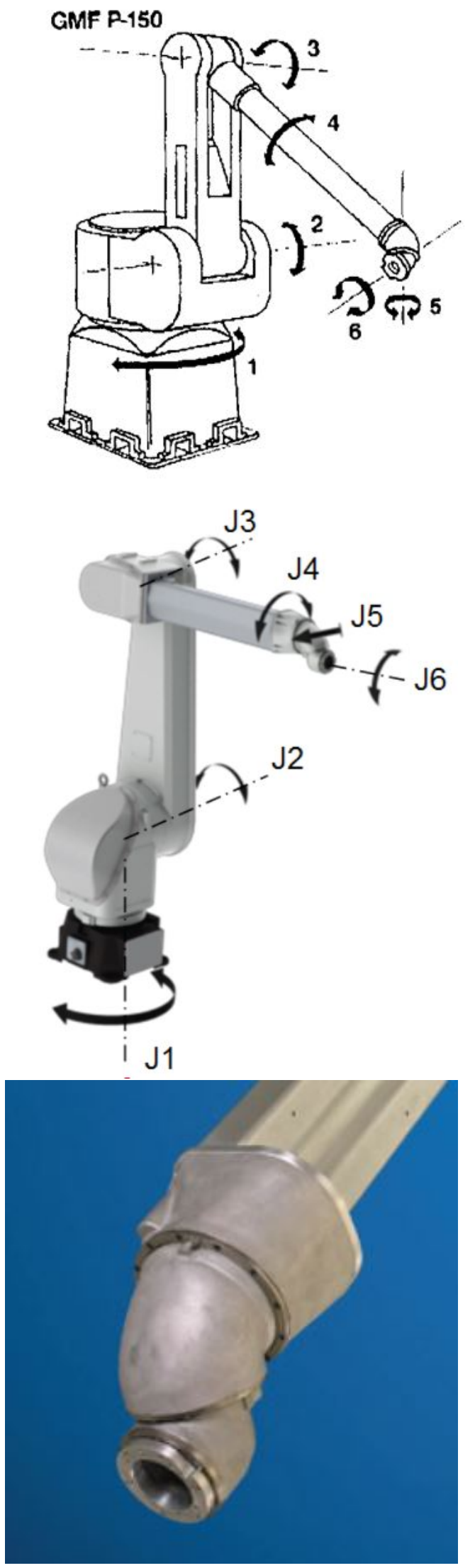

Figure 22 - Deux robots industriels $6 \mathrm{R}$ avec offset au poignet : le robot GMF P150 (en haut) et le robot Fanuc P250iB (au centre). Détail sur le poignet à offset (en bas).
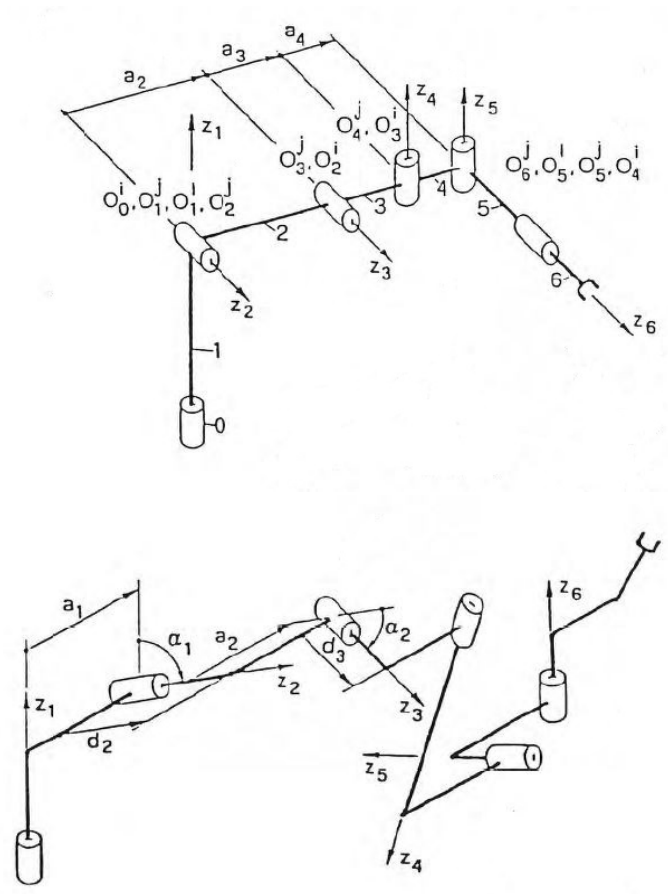

Figure 23 - Les deux robots $6 \mathrm{R}$ cuspidaux d'Innocenti [PI88]

d'une posture à l'autre.

\subsection{Règles de conception d'un robot}

On a vu qu'un robot cuspidal est plus difficile à mettre en oeuvre, en particulier pour l'étude de faisabilité de trajectoires. De plus, les postures d'un robot cuspidal ne peuvent pas être identifiées facilement. En effet, il n'existe généralement pas de critère simple permettant à un utilisateur de les reconnaître.

Il est donc plus raisonnable de chercher à concevoir un robot non cuspidal. Pour cela, on peut faire appel aux conditions énoncées dans le paragraphe 4.1 , et ajouter un poignet rotule si on désire concevoir un robot à 6 articulations. En appliquant ces règles, on retrouve la plupart des architectures des robots industriels courants, sauf la condition 6 du paragraphe 4.1. Cette condition produit des robots orthogonaux, inexistants sur le marché actuel. Cette classe de robots constitue pourtant une alternative intéressante aux robots anthropomorphes. En effet, en choisissant bien les paramètres géométriques, on peut obtenir un espace de travail compact et régulier [ZWC07a] avec des performances dynamiques meilleures qu'un robot anthropomorphe [NBW12]. 
A retenir : Certaines conditions géométriques comme deux axes parallèles ou sécants produisent des architectures de robots non cuspidales. II est possible d'établir une classification complète des robots $3 R$ orthgonaux en fonction du nombre de leurs points cusp. On obtient alors des conditions nécessaires et suffisantes sur les paramètres géométriques pour que le robot soit cuspidal ou non. Pour les robots $6 \mathrm{R}$ à poignet rotule, les conditions de cuspidalité sont celles du porteur. Par ailleurs, l'étude de plusieurs exemples a montré que lorsqu'un décalage d'axe est introduit sur un poignet rotule, le robot devient cuspidal. La classification des robots $6 \mathrm{R}$ quelconques est un problème qui reste largement ouvert.

\section{5 Étude de quelques robots paral- lèles cuspidaux}

Les paragraphes précédents ont été consacrés à l'étude des robots sériels. L'étude des robots parallèles, d'architecture plus complexe (voir paragraphe 1.1), nécessite d'étendre plusieurs définitions à cette classe de robot. Par ailleurs, la détermination du modèle géométrique direct et des singularités des robots parallèles est bien plus délicate que pour leurs homologues sériels. II n'existe pas de classification exhaustive portant sur une famille de robots parallèles comme c'est le cas pour les robots sériels 3R. Dans cet article, nous présentons quelques résultats pour quatre familles de robots parallèles : robots RPRPR, robots 3-RPR, robots 3-PPPS, et robots sphériques $2-U P S-U$. Les lettres $U$ et $S$ désignent une articulation de type cardan (universal joint en anglais) et sphérique. On rappelle que les lettres $R$ et $P$ désignent une articulation de type rotoïde et prismatique, respectivement. Une lettre soulignée signifie que l'articulation est motorisée.

\subsection{Les aspects pour les robots parallèles}

La notion d'aspects a été étendue aux robots parallèles avec une seule solution au modèle géométrique inverse dans [WC97] puis aux robots parallèles avec plusieurs solutions au modèle géométrique inverse dans [CW98]. Dans cet article, seuls les robots parallèles n'ayant qu'une seule solution géométrique inverse sont analysés. On rappelle ci-dessous la définition étendue des aspects pour ces robots parallèles.

Les Aspects pour les robots parallèles : on appelle aspects $\mathcal{W} \mathcal{A}_{j}$ d'un robot parallèle, les plus grands domaines de l'espace de travail $\mathcal{W}$ tels que :

$-\mathcal{W} \mathcal{A}_{j} \in \mathcal{W}$

- $\mathcal{W} \mathcal{A}_{j}$ est connexe;

$-\forall X \in \mathcal{W}, \operatorname{det}(\mathbf{A}) \neq \mathbf{0}$ où $\mathbf{A}$ est la matrice jacobienne parallèle du robot, c'est-à-dire associée à l'opérateur géométrique inverse $\mathbf{g}$ défini par $\mathbf{q}=$ $\mathbf{g}(\mathbf{X})$.

En d'autres termes, les aspects sont les plus grands domaines de l'espace de travail exempts de toute singularité parallèle. L'ensemble des aspects $\left\{\mathcal{W} \mathcal{A}_{j}\right\}$ peut être obtenu en soustrayant de l'espace de travail les configurations singulières $\mathcal{S}$ et en effectuant une analyse de connexité : $\left\{\mathcal{W} \mathcal{A}_{j}\right\}=\mathrm{CC}\{\mathcal{W}-\mathcal{S}\}$ où $\mathrm{CC}=$ composantes connexes et où - désigne la différence entre ensembles.

\section{Exemple 5 : le robot parallèle plan RPRPR}

La figure 24 présente un robot parallèle plan à deux degrés de liberté. Ses variables d'entrée sont les longueurs des articulations prismatiques $\rho_{1}$ et $\rho_{2}$, et ses variables de sortie sont les deux coordonnées de position $(x, y)$ du point $P$. En écrivant que le point $P$ se situe à l'intersection de deux cercles, l'un centré en $A$ et de rayon $\rho_{1}$, l'autre centré en $B$ et de rayon $\rho_{2}$, a obtient les deux équations de contraintes suivantes:

$$
\begin{aligned}
& \rho_{1}^{2}=x^{2}+y^{2} \\
& \rho_{2}^{2}=(x-l)^{2}+y^{2}
\end{aligned}
$$

où $/$ désigne la distance entre les deux pivots $A$ et $B$. Par ailleurs, les butées articulaires produisent deux contraintes supplémentaires données par les inéquations $\rho_{i \min } \leq \rho_{i} \leq \rho_{i \max }$ avec $i=(1,2)$. La figure 26 représente un espace de travail divisé en deux aspects. Comme ce robot ne possède que deux modes d'assemblage (le point $P$ est au dessus ou au dessous de l'axe des $x$ ), les aspects sont des domaines d'unicité du modèle géométrique direct et ce robot n'est donc pas cuspidal.

En faisant l'hypothèse que $\rho_{i}$ min $>0$ (ce qui est en général le cas pour des raisons technologiques), le robot n'a pas de singularité sérielle et ne possède qu'une seule solution au modèle géométrique inverse. Les singularités parallèles de ce robot sont les configurations pour lesquelles les points $A, B$ et $P$ sont alignés (figure 25 ). D'après la figure 26 , il existe deux aspects $\mathcal{W} \mathcal{A}_{1}$ et $\mathcal{W} \mathcal{A}_{2}$. On voit que par rapport aux robots sériels, les aspects des robots parallèles divisent l'espace de travail et non pas le domaine articulaire. La trajectoire $C D$ est réalisable dans l'aspect $\mathcal{W} \mathcal{A}_{1}$ tandis que la trajectoire $E F$, à cheval entre les deux aspects $\mathcal{W} \mathcal{A}_{1}$ et $\mathcal{W} \mathcal{A}_{2}$, ne peut pas être suivie de manière continue sans rencontrer de configuration singulière.

\subsection{Robot parallèle cuspidal}

Robot parallèle cuspidal : Un robot parallèle est dit cuspidal s'il peut changer de mode d'assemblage, c'est-àdire changer de solution du modèle géométrique direct, sans passer par une singularité.

\section{Exemple 6 : le robot parallèle plan 3-RPR}

La figure 27 montre un robot parallèle plan à trois degrés de liberté. Ses variables d'entrée sont les longueurs des articulations prismatiques $\rho_{1}, \rho_{2}$ et $\rho_{3}$, et ses variables de sortie sont les deux coordonnées de position $(x, y)$ du point $B_{1}$ et l'orientation $\phi$ de la plate-forme mobile. Les paramètres géomé- 


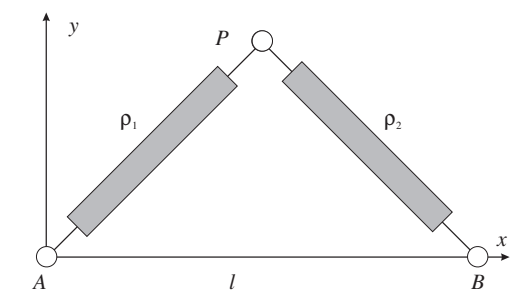

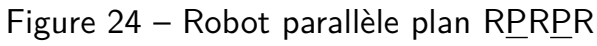

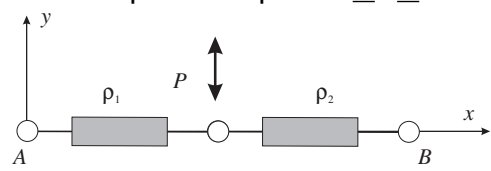

Figure 25 - Singularité du robot parallèle plan RPRPR

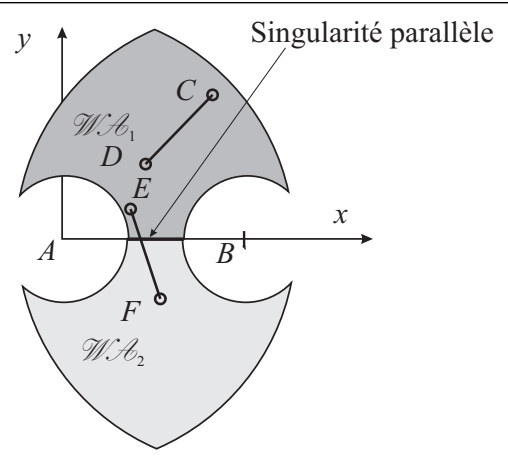

Figure 26 - Les deux aspects du robot parallèle plan RPRPR pour $I=5, \rho_{\min }=2$ et $\rho_{\max }=7$

triques sont les trois côtés de la plate-forme mobile $I_{1}, I_{2}, I_{3}$ (avec $\theta$ positif) et la position des centres de rotation de la base définis par $A_{1}, A_{2}$ et $A_{3}$. Le repère de référence est centré sur $A_{1}$ et l'axe des $x$ passe par $A_{2}$. Ainsi, $A_{1}=(0,0), A_{2}=\left(c_{2}, 0\right)$ et $A_{3}=\left(c_{3}, d_{3}\right)$

La mise en équations de ce robot est donnée dans [CJR92] :

$$
\begin{aligned}
\rho_{1}^{2}= & x^{2}+y^{2} \\
\rho_{2}^{2}= & \left(x+l_{2} \cos (\phi)-c_{2}\right)^{2}+\left(y+l_{2} \sin (\phi)\right)^{2} \\
\rho_{3}^{2}= & \left(x+l_{3} \cos (\phi+\theta)-c_{3}\right)^{2}+ \\
& \left(y+l_{3} \sin (\phi+\theta)-d_{3}\right)^{2}
\end{aligned}
$$

Nous ne détaillons pas le calcul du modèle géométrique inverse qui conduit à la résolution d'un polynôme de degré 6 , celui-ci est décrit par exemple dans [Mer97]. Pour notre étude, nous prendrons les mêmes dimensions que dans [IP98] et [Mer97] (Tableau 1).

Les singularités du robot 3-R $\underline{P R}$ sont :

- Les singularités parallèles : de telles configurations sont atteintes lorsque l'axe passant par les trois articulations prismatiques se croisent en un seul point (Figure 28) ou

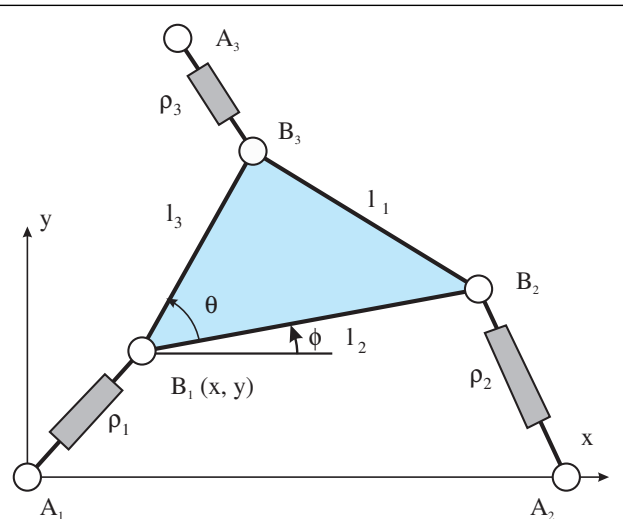

Figure 27 - Un robot parallèle de type 3-RPR

Tableau 1 - Dimensions du robot parallèle plan 3-RPR étudié (unités sans importance)

\begin{tabular}{|c|c|}
\hline$A_{1}=(0,0)$ & $I_{1}=B_{2} B_{3}=16.5$ \\
$A_{2}=(15.9,0)$ & $I_{2}=B_{1} B_{2}=17$ \\
$A_{3}=(0,10)$ & $I_{3}=B_{3} B_{1}=20.8$ \\
\hline
\end{tabular}

sont parallèles ;

- Les singularités sérielles : elles correspondent aux configurations pour lesquelles: $\rho_{1}=0$ ou $\rho_{2}=0$ ou $\rho_{3}=0$. En pratique, comme pour le robot précédent, ces conditions ne peuvent généralement pas être remplies et c'est ce qui sera supposé dans cet exemple.

Les limites des articulations prismatiques sont celles choisies par [IP98] :

$$
10.0 \leq \rho_{i} \leq 32.0 \text { pour } \quad i=(1,2,3)
$$

Les unités n'ont pas d'importance dans cet exemple. La figure 29 représente l'espace de travail dont les frontières sont obtenues par l'intersection des surfaces générées par les limites articulaires.

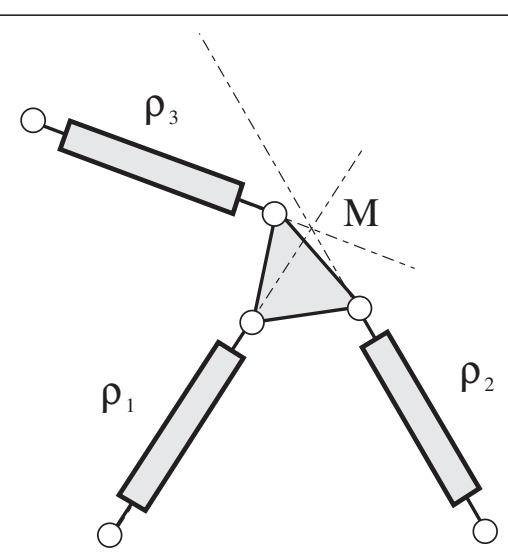

Figure 28 - Singularité parallèle du robot parallèle 3-RPR 


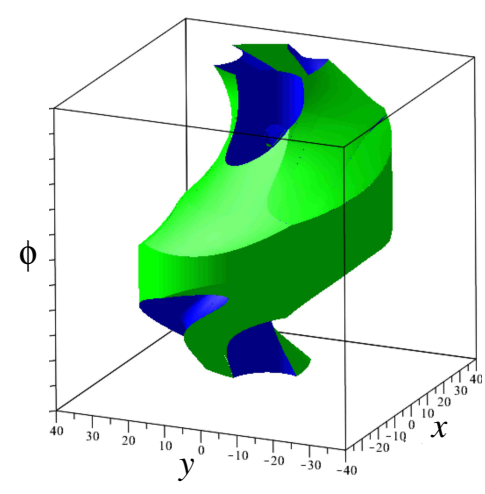

Figure 29 - Espace de travail du robot 3-RPR avec les limites articulaires

Lorsque $\rho_{1}=15.0, \rho_{2}=15.4$ et $\rho_{3}=12.0$, le robot admet 6 solutions au modèle géométrique direct soit 6 modes d'assemblage (Tableau 2) (Figure 30).

Tableau 2 - Les 6 modes d'assemblage du robot 3-RPR pour $\rho_{1}=15.0, \rho_{2}=15.4$ et $\rho_{3}=12.0$ ( $\phi$ donné en radians)

\begin{tabular}{|c|c|c|c|}
\hline & $x$ & $y$ & $\phi$ \\
1 & -8.715 & 12.183 & -0.987 \\
2 & -5.495 & -13.935 & -0.047 \\
3 & -14.894 & 1.596 & 0.244 \\
4 & -13.417 & -6.660 & 0.585 \\
5 & 14.920 & -1.337 & 1.001 \\
6 & 14.673 & -3.013 & 2.133 \\
\hline
\end{tabular}

Une trajectoire de changement de mode d'assemblage est proposée par [IP92]. La Figure 30 représente cette trajectoire dans l'espace articulaire pour $\rho_{1}=15, \rho_{2}=15.4$ et $\rho_{3}=12$. Les modes d'assemblage -2-,-3-, -6- du tableau 2 sont dans le même aspect $\left(\mathcal{W} \mathcal{A}_{1}\right)$ (Figure 31$)$ et les modes d'assemblage -1-, -4- et -5- sont dans un second aspect $\left(\mathcal{W A}_{2}\right)$. Cela signifie que les aspects ne sont pas des domaines d'unicité pour le modèle géométrique direct.

La figure 32 représente les surfaces de singularités dans l'espace de travail avec la trajectoire de changement d'assemblage non singulière (en haut) et une trajectoire de changement de mode d'assemblage singulière (en bas). L'analyse des trajectoires a été présentée dans [ZWC08] pour le robot plan 3-RPR et dans $[\mathrm{Cha}+]$ pour un robot sphérique de type 2-UPS-U.

Une analyse de l'espace articulaire de ce robot est présenté dans [ZWC07b] en réalisant des coupes en fonction de $\rho_{1}$. La figure 33 montre une coupe de l'espace articulaire dans le plan $\left(\rho_{2}, \rho_{3}\right)$ en $\rho_{1}=17$. II y a 5 points cusps (entourés par des losanges $\diamond$ ) et les singularités font apparaitre des régions avec 2, 4 ou 6 modes d'assemblage.

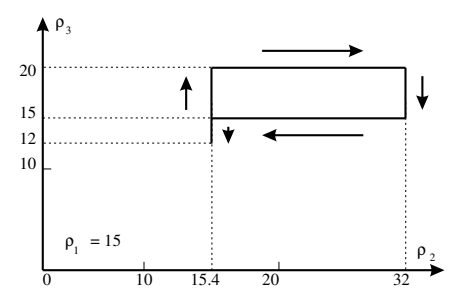

Figure 30 - Exemple de trajectoire de changement de mode d'assemblage du robot parallèle 3-RPR dans l'espace articulaire pour $\rho_{1}=15$

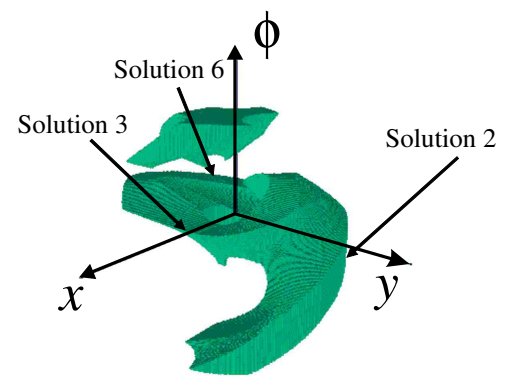

Figure 31 - Modes d'assemblage 2-3-6 dans l'aspect $\mathcal{W} \mathcal{A}_{1}$ du robot parallèle $3-\mathrm{R} \underline{\mathrm{PR}}$ dans l'espace de travail

\subsection{Identifier les robots parallèles cuspi- daux}

Un robot rencontre une singularité parallèle lorsque la matrice Jacobienne parallèle n'est plus de rang plein. De plus, deux modes d'assemblage coïncident sur une singularité parallèle. Lorsque trois modes d'assemblage fusionnent, on dit que le robot est dans une configuration cuspidale. Ainsi, une configuration cuspidale peut être caractérisée par une racine triple du polynôme du modèle géométrique direct. McAree et Daniel montrent que dans de telles configurations, le robot perd les contraintes de premier et de second ordre [MD99]. En pratique, cela signifie que le robot est très instable dans une configuration cuspidale.

La configuration $P_{0}=(\mathbf{q}, \mathbf{X})$ d'un robot est dite cuspidale si et seulement si $\mathbf{X}$ est une solution de multiplicité 3 [Mor+10].

Dans [Mor+10], la notion de variété discriminante et une généralisation du critère jacobien permettent une description complète et certifiée des configurations cuspidales. Cette méthode a été implémentée dans la librairie SIROPA du logiciel de calcul formel Maple [Cha+19].

\subsection{Classification des robots parallèles}

II existe peu d'études portant sur la classification des robots parallèles. On peut noter les travaux de Merlet [Mer96] sur les robots parallèles plans où le nombre maximum de solutions au modèle géométrique direct est donné en fonction de l'architecture des jambes et de la position de l'articulation motorisée (RRR, RPR, 


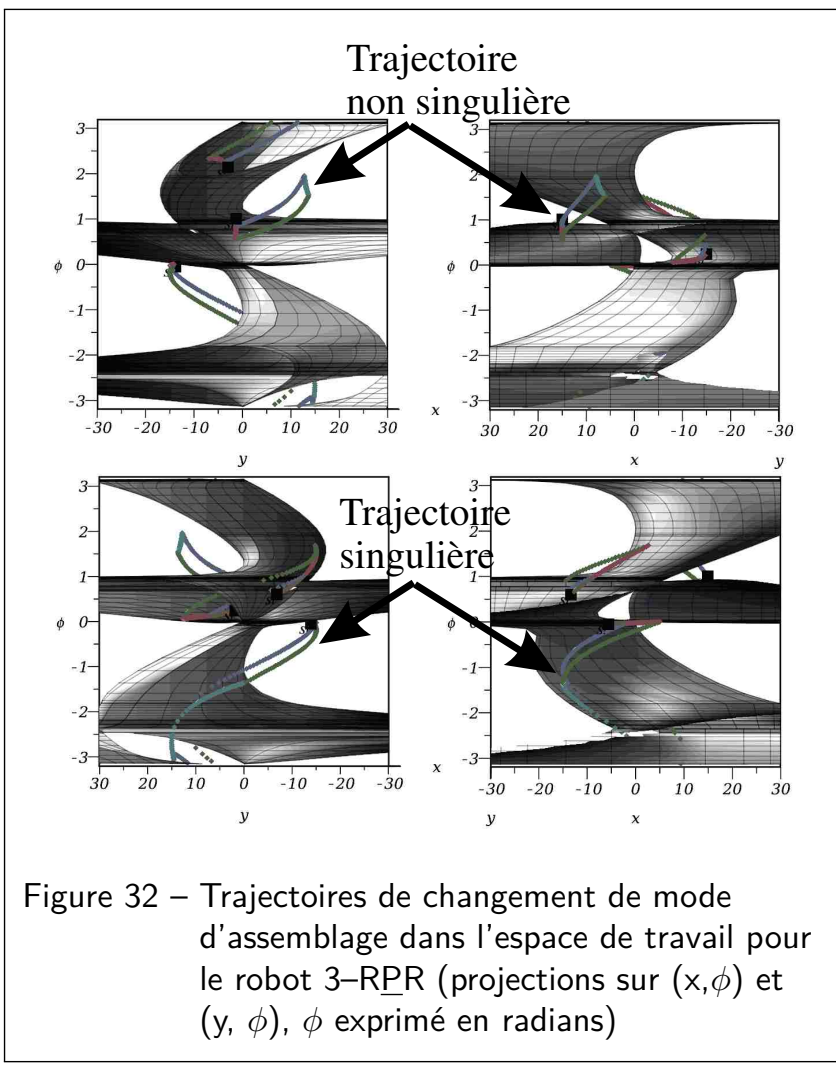

Figure 33 - Coupe de l'espace articulaire du robot
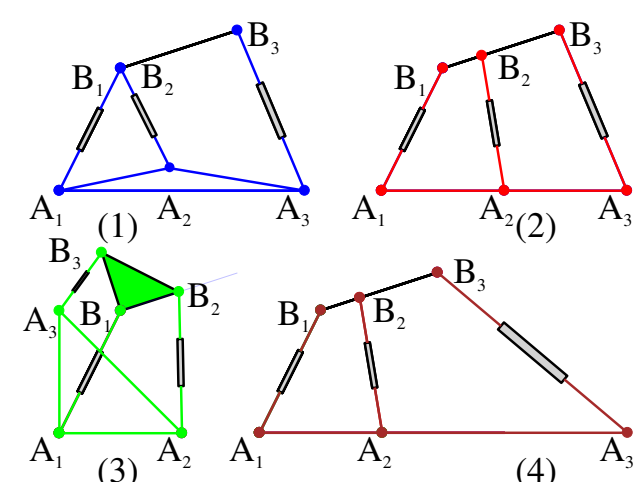

(4)

Figure 34 - Exemples de robots 3-RPR analytiques de types 1, 2, 3 et 4

PRR, PPR, RRP, RPP et PRP). Pour les robots parallèles plans, il peut exister 2,4 , ou 6 solutions au modèle géométrique direct.

Pour les robots $3-R P R$, des conditions géométriques existent pour avoir un modèle géométrique direct analytique, c'est-à-dire qui se résout avec des équations du deuxième ou troisième degré.

Quatre familles de robots analytiques ont été trouvées [KG00] :

- Type 1 : Les robots pour lesquels deux des articulations de la base ou de la plate-forme mobile coïncident (Fig. 34, en haut à gauche) ;

- Type 2 : Les robots dont la plate-forme et la base sont alignées et quelconques (Fig. 34, en haut à droite);

- Type 3 : Les robots dont la plate-forme et la base forment des triangles semblables, en bas à gauche);

- Type 4 : Les robots dont la plate-forme et la base sont alignées et semblables (Fig. 34, en bas à droite).

Pour les types 1,3 , et 4 , le modèle géométrique direct se résout avec des équations quadratiques en série. Ces robots ne peuvent donc pas présenter de configuration cuspidale. Même si l'absence de configuration cuspidale ne signifie pas que le robot est non cuspidal en général, il semble que ce soit le cas pour les robots de type 1, 3 et 4 . Pour le type 2 , on a à résoudre une équation $\mathrm{cu}-$ bique puis une équation quadratique. Le robot peut donc avoir des configurations cuspidales et 2 modes d'assemblage dans un même aspect issu de la résolution de la cubique. Cependant, même si, à notre connaissance, aucune étude n'a été réalisée pour classifier cette famille de robots parallèles, tous les robots $3-R \underline{P} R$ de type 2 qui ont été étudiés dans la littérature ont au plus 4 modes d'assemblage et sont cuspidaux.

Une autre famille de robots analytiques est décrite dans [WCZ07]. Pour ces robots, la base et la plate-forme forment des triangles égaux mais l'un est retourné par rapport à l'autre (rotation de 180 degrés autour d'un des côtés). Leur modèle géométrique direct se résout avec un polynôme cubique puis un polynôme quadratique en série comme pour les robots de type 2 décrits plus haut. 


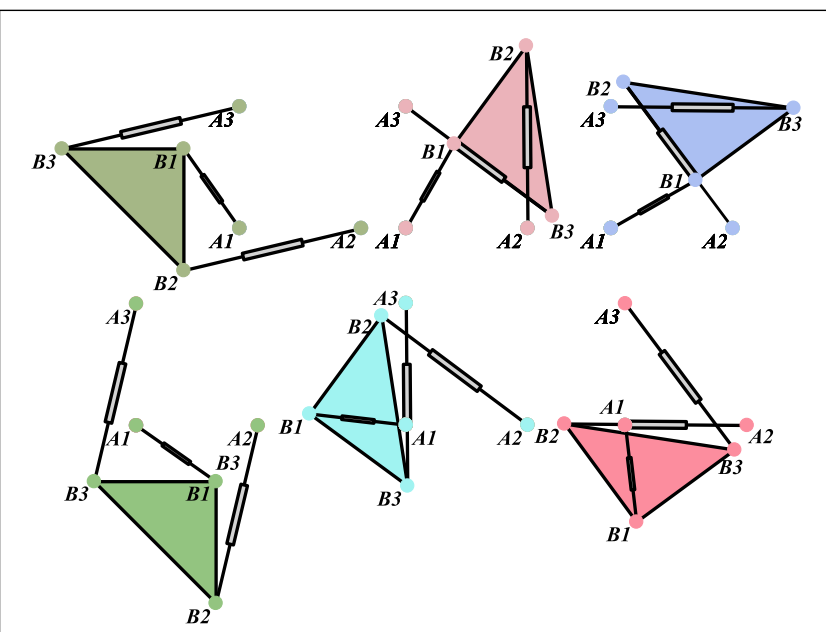

Figure 35 - Les 6 modes d'assemblage d'un robot 3-RPR analytique avec base et plate-forme formant des triangles égaux mais dont l'un est retourné par rapport à l'autre.

II est donc aussi simple que pour ces derniers. Cependant, ces robots possèdent des configurations cuspidales et sont donc cuspidaux [CWC11]. Lorsque la plate-forme est proche du triangle de la base, les jambes peuvent se croiser (voir Fig. 35), ce qui oblige le concepteur de ces robots à positionner les jambes dans des plans décalés.

\subsection{Faisabilité de trajectoires pour les ro- bots parallèles}

La notion de parcourabilité pour les robots parallèles est importante et s'analyse directement dans l'espace de travail. En effet, les singularités parallèles découpent l'espace de travail en plusieurs régions distinctes, qui sont les aspects.

Région t-parcourable pour un robot parallèle : Une région de l'espace de travail est dite t-parcourable si toute trajectoire continue dans cette région peut être parcourue par l'organe terminal sans jamais rencontrer une singularité parallèle. Pour un robot parallèle, les régions t-parcourables sont les aspects [CW99].

Si les aspects sont les régions t-parcourables, deux questions se posent :

- Quel est l'impact des changements de mode d'assemblage sur la sécurité lors de la planification de trajectoires dans un aspect lorsque le robot est cuspidal?

- Les régions t-parcourables sont-elles plus grandes si le robot parallèle est cuspidal ?

\subsubsection{Impact des changements de mode d'assem- blage}

Lorsqu'un robot parallèle est cuspidal, il peut changer de mode d'assemblage sans rencontrer de configuration singulière dans une région t-parcourable. Cela signifie que les modes d'assemblage au point de départ et au

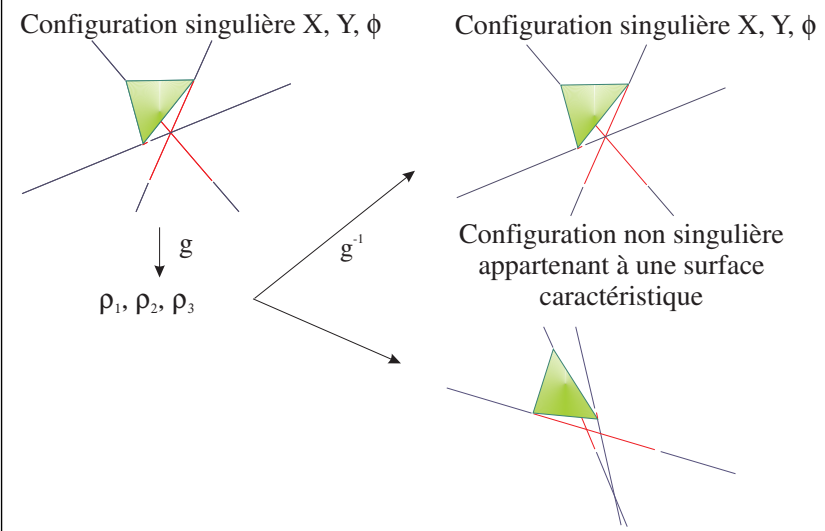

Figure 36 - Construction des surfaces caractéristiques pour les robots parallèles

point d'arrivée d'une trajectoire peuvent être différents, alors que la commande qui ne connaît que les positions articulaires ne peut pas le détecter. Si le robot s'arrête et perd I'historique de ses mouvements, le mode d'assemblage courant peut être erroné. II est donc nécessaire, soit d'ajouter des capteurs sur des articulations passives judicieusement choisies permettant d'identifier le mode d'assemblage courant sans ambiguïté, soit de toujours rester dans un domaine d'unicité du modèle géométrique direct.

La notion de domaines d'unicité a été formulée pour les robots parallèles avec une ou plusieurs solutions au modèle géométrique inverse [CMW11]. Ici, seule la première formulation nous intéresse. Les domaines d'unicité répondent à la problématique de la sécurité en créant des régions où le robot ne change pas de mode d'assemblage. Comme pour les robots sériels, les domaines d'unicité nécessitent le calcul de surfaces caractéristiques qui séparent les solutions dans les aspects.

Les surfaces caractéristiques pour les robots parallèles: Soit $\mathcal{W} \mathcal{A}_{j}$ un aspect de l'espace de travail $W$. On définit les surfaces caractéristiques de l'aspect $\mathcal{W} \mathcal{A}_{j}$, notées $\mathcal{S}_{C}\left(\mathcal{W} \mathcal{A}_{j}\right)$, comme l'image réciproque dans $\mathcal{W} \mathcal{A}_{j}$ de l'image $\mathbf{g}\left(\overline{\mathcal{W}} \mathcal{A}_{j}\right)$ des frontières $\overline{\mathcal{W}}_{j}$ qui délimitent $\mathcal{W} \mathcal{A}_{j}$ où $\overline{\mathcal{W A}}_{j}$ est la frontière de l'aspect $\mathcal{W} \mathcal{A}_{j}$.

Pour chaque aspect, on considère les frontières qui correspondent aux configurations singulières et aux butées articulaires (Fig. 36). Le calcul des surfaces caractéristiques est difficile à réaliser lorsque le robot a plus de deux aspects. En effet, la frontière entre les aspects est commune lorsqu'il n'y a que deux aspects, alors qu'avec quatre aspects, il est nécessaire de distinguer les frontières, ce qui est très difficile algébriquement. Comme pour les robot sériels, les surfaces caractéristiques définissant des domaines d'unicité qui ne sont pas les plus grands, il faut alors procéder à la réunion de plusieurs de ces domaines adjacents (voir [Cha98] pour plus de détails).

\section{Exemple 7 : le robot parallèle plan RPR- 2RPR}

Ce robot parallèle est composé de trois jambes 


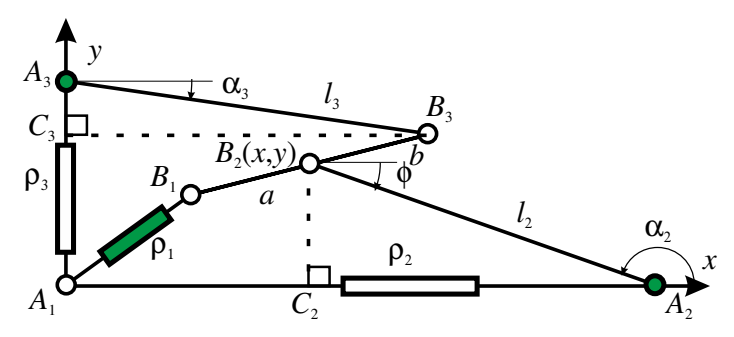

Figure 37 - Robot parallèle RPR-2RPR

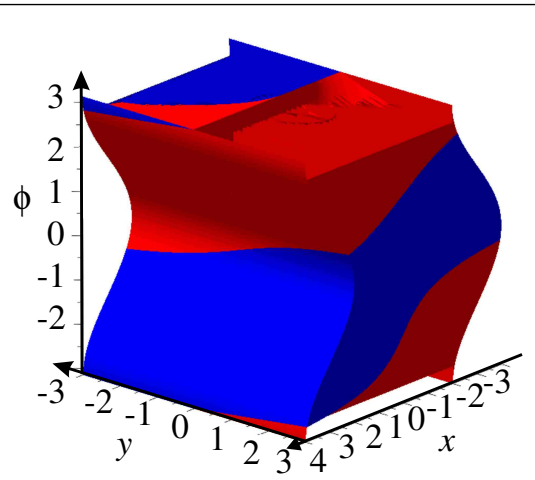

Figure 38 - Les deux aspects du robot parallèle RPR-2RPR

et d'une plate-forme mobile. La première jambe, de type RPR, est composée de 2 pivots passifs et d'une prismatique actionnée. Les 2 autres jambes, de type $\underline{R P R}$, sont composées d'une liaison pivot actionnée, d'une liaison prismatique et d'un pivot passifs. Les trois variables motorisées sont décrites $\operatorname{par} \rho_{1}, \alpha_{2}$ et $\alpha_{3}$. La plate-forme mobile possède 3 degrés de liberté définis par les coordonnées $[x$ y $\phi]$ (Figure 37). Les équations de contrainte sont les suivantes :

$$
\begin{aligned}
\rho_{2}+l_{2} \cos \left(\alpha_{2}\right)-x & =0 \\
l_{2} \sin \left(\alpha_{2}\right)-y & =0 \\
(x-a \cos (\phi))^{2}+(y-a \sin (\phi))^{2}-\rho_{1}^{2} & =0 \\
l_{3} \cos \left(\alpha_{3}\right)-b \cos (\phi)-x & =0 \\
\rho_{3}+l_{3} \sin \left(\alpha_{3}\right)-b \sin (\phi)-y & =0
\end{aligned}
$$

L'espace de travail de ce robot est composé de deux aspects dans lesquels le nombre de modes d'assemblage est 2 ou 4 [CMW11] (Figure 38). On peut reconnaître dans une coupe de l'espace articulaire pour $\alpha_{2}=\arcsin 1 / 6$, la présence de 4 points cusps sur la Figure 39. Le calcul des surfaces caractéristiques de ce robot est simple car il n'existe que deux aspects. Les surfaces caractéristiques sont tangentes aux singularités (Fig. 40) et le point de tangence est l'image d'un point cusp. On observe les mêmes propriétés que pour les robots sériels mais en inversant l'espace de travail et l'espace articulaire. La figure 41 représente les 4 domaines d'unicité de la figure 38 .
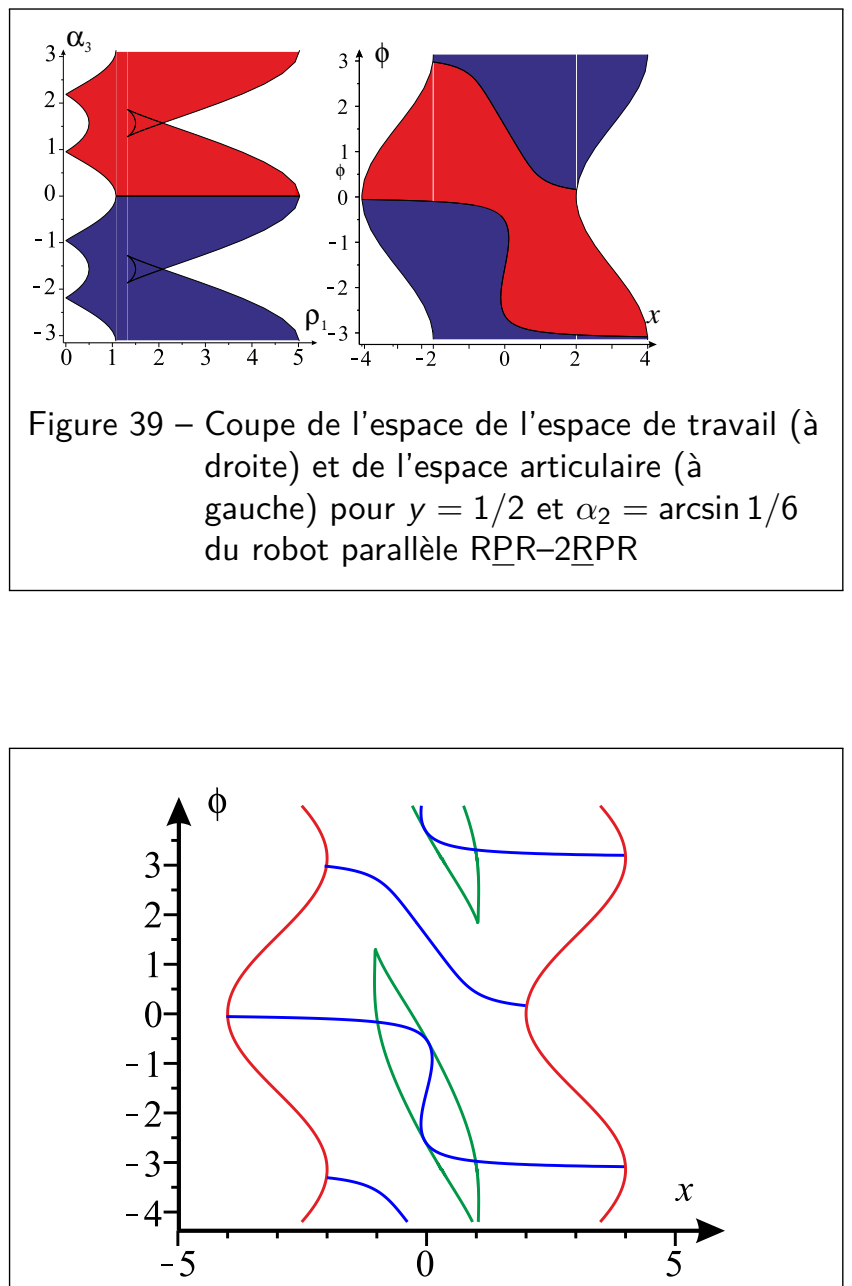

Figure 40 - Singularités (en bleu) et surfaces caractéristique (en vert) du robot parallèle RPR-2RPR
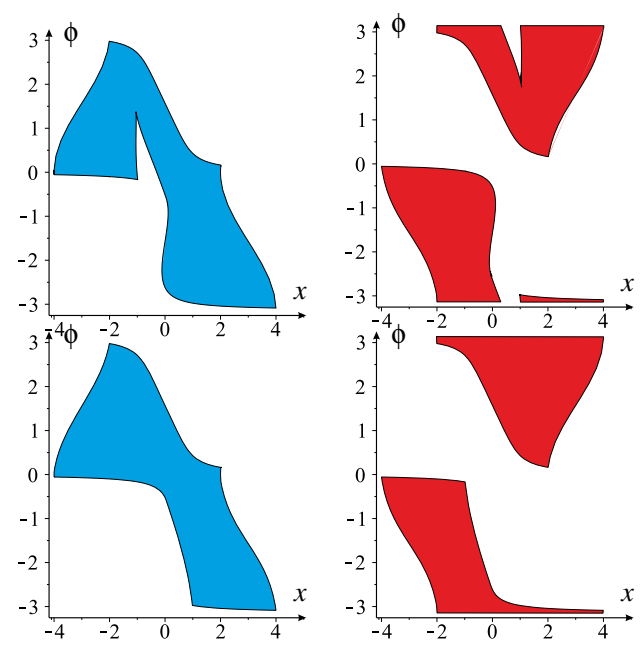

Figure 41 - Domaines d'unicité du robot parallèle RPR-2RPR 


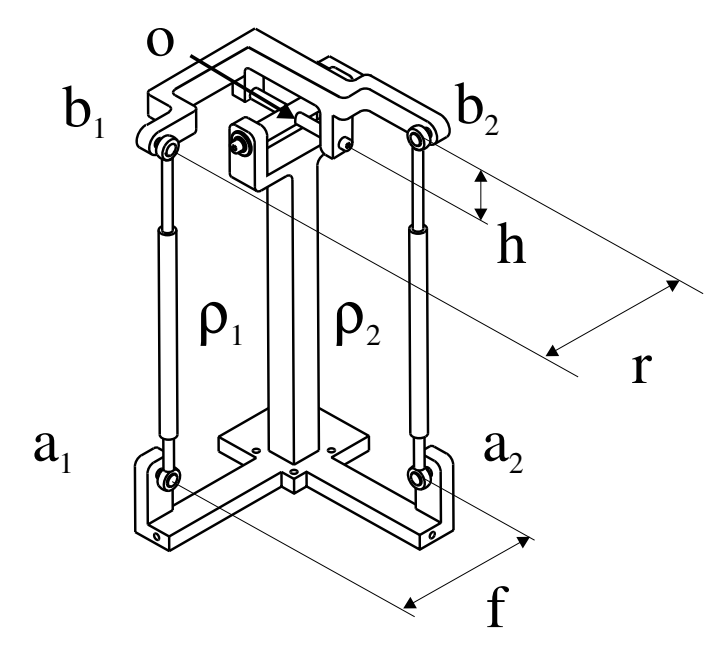

Figure 42 - Robot parallèle sphérique 2-UPS-U

\section{Exemple 8 : le robot parallèle sphérique 2- UPS-U}

Ce robot parallèle sphérique est composé de trois jambes et d'une plate-forme mobile. Les deux premières jambes, U $\underline{P} S$, sont composés d'un cardan, d'une prismatique actionnée et d'une liaison sphérique. La dernière jambe est passive (non actionnée) et est constituée d'un cardan qui limite la mobilité de la plate-forme mobile à 2 degrés de liberté définis par les rotations $\alpha$ et $\beta$ (Figure 42). Les deux articulations prismatiques sont actionnées et définies $\mathrm{p}$ ar $\rho_{1}$ et $\rho_{2}$. L es é quations de contraintes s'écrivent :

$$
\begin{aligned}
-2(f h+\cos \alpha r) \sin \beta & +2(h \cos \alpha-f r) \cos \beta \\
+f^{2} & +h^{2}+r^{2}+1=\rho_{1}^{2} \\
2 h(f \sin \alpha+\cos \alpha) \cos \beta & -2 f \cos \alpha r+2 \sin \alpha r \\
+f^{2} & +h^{2}+r^{2}+1=\rho_{2}^{2}
\end{aligned}
$$

En fonction des paramètres de conception, le nombre d'aspects ainsi que le nombre de solutions par aspect changent. Les figures 43 et 44 présentent deux exemples d'espace de travail avec une décomposition en aspects. Pour les paramètres du robot de la figure 43 , les équations liées aux singularités se factorisant, le robot a 4 modes d'assemblages et 4 aspects (chaque couleur es associée à un aspect) et il n'y a qu'une solution au modèle géométrique direct par aspect : le robot n'est donc pas cuspidal. Pour les paramètres du robot de la figure 44 , le robot a 4 aspects mais admet jusqu'à 6 modes d'assemblage. De plus, on montre que dans l'aspect en jaune, il peut y avoir jusqu'à trois solutions : le robot est donc cuspidal.

\subsubsection{Taille des régions $t$-parcourables}

Lorsqu' un robot parallèle non cuspidal admet $n$ solutions à son modèle géométrique direct, l'espace de travail est

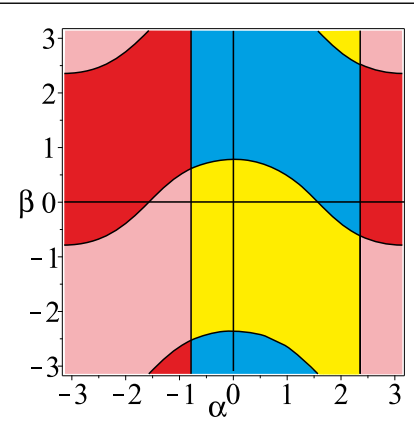

Figure 43 - Espace de travail du robot parallèle sphérique 2-UPS-U pour $h=0, r=1$, $f=1$ où chaque couleur est associé à un aspect (4 modes d'assemblage) 
divisé en un minimum de $n$ aspects (si les limites articulaires le permettent). Inversement, un robot cuspidal a plusieurs solutions à son modèle géométrique direct dans un même aspect. II est alors possible d'avoir des régions t-parcourables plus vastes. Cette question a été étudiée pour la première fois dans [WC98] à l'aide d'un robot parallèle plan $3-\mathrm{R} \underline{\mathrm{PR}}$.

\section{Exemple 9 : le robot parallèle sphérique 2- UPS-U}

Les robots des Figures 43 et 44 ont le même nombre d'aspects mais pour celui qui est cuspidal, les aspects représentés en jaune et en orange sont plus grands.

Plus particulièrement, l'aspect représenté en jaune autour de la position d'origine $(\alpha=0, \beta=0)$ de la figure 44 e st $\mathrm{p}$ lus $\mathrm{g}$ rand q ue c elui l ocalisé au même endroit de la figure 43. Il est d onc plus intéressant pour l'utilisateur. Cependant, pour assurer la sécurité sans ajouter de capteurs supplémentaires, il est nécessaire de savoir si le robot change de mode d'assemblage lors de ces mouvements. Pour ce robot parallèle sphérique, [Cha+20] et [Cha+21] ont étudié la position des singularités et des surfaces caractéristiques pour que le robot ne change pas de mode d'assemblage à l'intérieur d'un espace de travail prescrit (figure 45). Cet espace de travail représente le besoin de l'utilisateur en fonction de son application.

La figure 45 r eprésente I 'espace d e t ravail avec les surfaces caractéristiques. Un espace de travail prescrit $\alpha=\left[\begin{array}{ll}-1 & 1\end{array}\right]$ et $\beta=\left[\begin{array}{ll}-1 & 1\end{array}\right]$ est localisé autour de la position d'origine $(\alpha=0, \beta=0)$. L'image de la frontière de cet espace de travail prescrit est représenté sur la figure 46 . I l a été démontré que cette trajectoire contourne bien des points cusps $C_{4}, C_{5}$ et $C_{6}$ mais ne provoque pas de changement de mode d'assemblage. Pour un autre robot ou pour un autre espace de travail prescrit, la conclusion aurait pu être différente.

\subsection{Cas des robots à 6 degrés de liberté}

L'étude des robots parallèles à 6 degrés de liberté est un problème difficile lorsque les degrés de liberté de translation et de rotation ne sont pas découplés, ce qui est le cas le plus fréquent. Dans ce cas, les méthodes algébriques arrivent à leurs limites de résolution et il est nécessaire de recourir à des méthodes numériques (par exemple, discrétisation régulière de l'espace ou utilisation de méthodes basées sur l'analyse par intervalles). L'exemple suivant est l'un des rares robots parallèles découplés et pouvant être étudié complètement avec des outils algébriques.

\section{Exemple 10 : Le robot parallèle 3-PPPS or-} thogonal.

Ce robot (figure 47) p ossède 6 d egrés d e liberté [CWC12] et les jambes sont constituées de trois articulations prismatiques placées orthogonalement, deux articulations actionnées suivies d'une passive

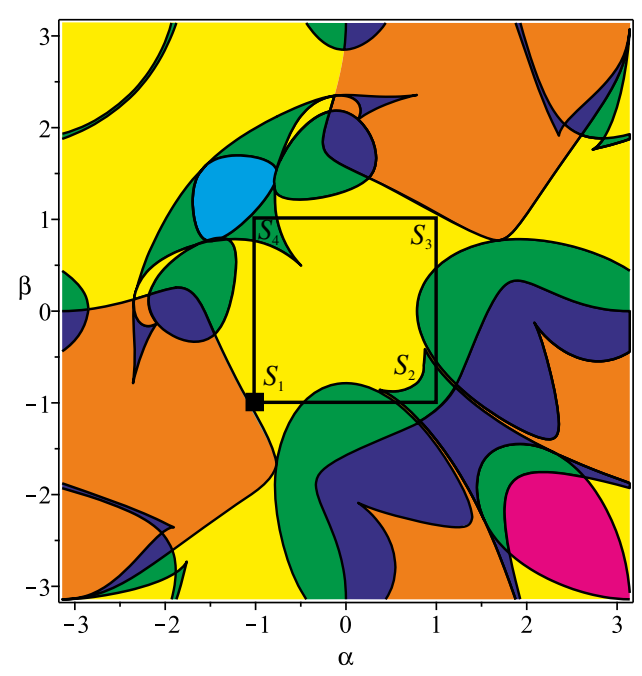

Figure 45 - Décomposition de l'espace de travail du robot de la figure 44 avec les surfaces caractéristiques et la frontière de l'espace de travail prescrit $\alpha=\left[\begin{array}{ll}-1 & 1\end{array}\right]$ et $\beta=\left[\begin{array}{ll}-1 & 1\end{array}\right]$

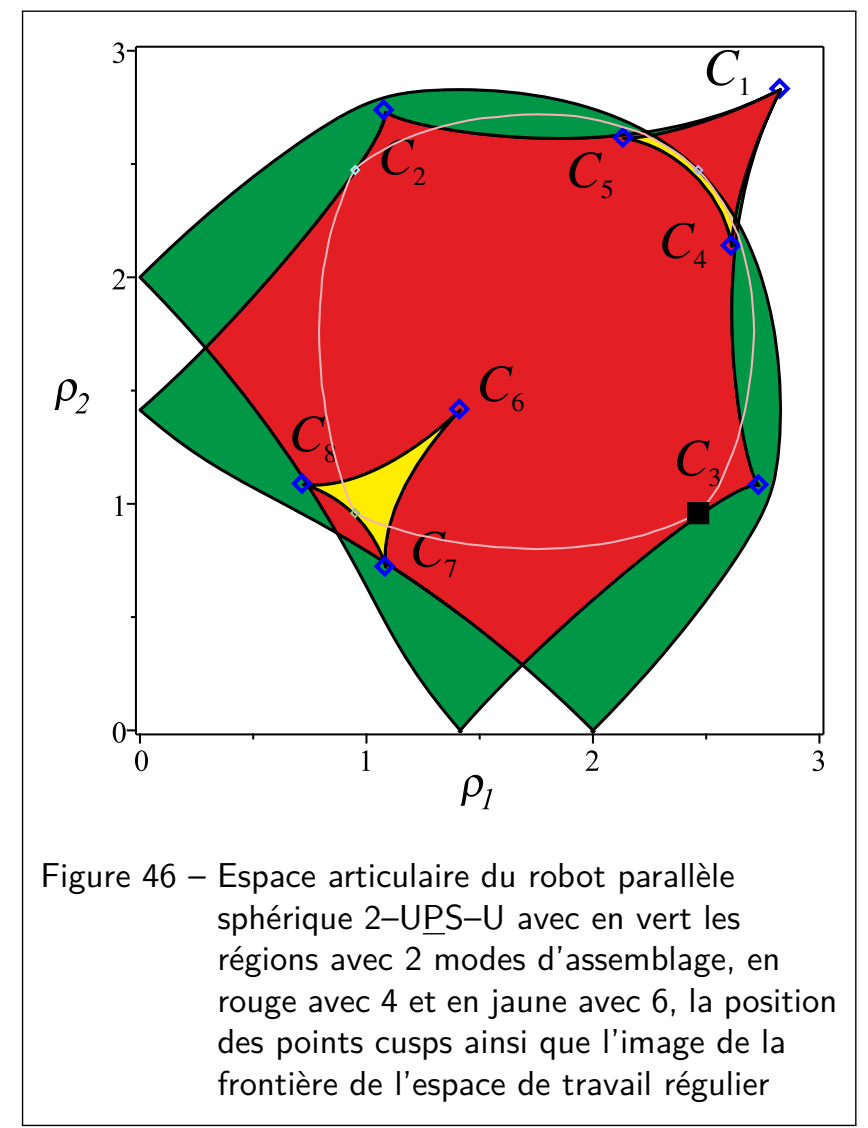


et d'une liaison rotule. On peut montrer que le lieu des singularités est indépendant de la position et est donc fonction uniquement de l'orientation. En effet, par un changement de variable judicieux, il est possible d'étudier l'espace articulaire de ce robot avec seulement trois liaisons prismatiques décrites par $(x, y, z)$. L'espace de travail peut être décrit avec trois angles d'Euler modifiés ( $\phi$ : azimuth, $\theta$ : tilt et $\sigma$ : torsion) [BZG02].

Ainsi, l'équation des singularités peut s'écrire à l'aide d'un produit de 3 facteurs :

$$
\begin{aligned}
& \left(\sqrt{2} \sin (3 \phi-\sigma)\left(\sin \left(\frac{\theta}{2}\right)\right)^{3}-\cos \left(\frac{3 \theta}{2}\right) \cos (\sigma)\right) \\
& \sin \left(\frac{\theta}{2}\right)\left(\cos \left(\frac{\theta}{2}\right)\right)^{2}=0
\end{aligned}
$$

On obtient alors une surface qui divise l'espace de travail en deux aspects.

La Figure 48 représente les singularités dans l'espace de travail ainsi qu'un exemple de trajectoire non singulière qui relie 4 modes d'assemblage pour $x=9 / 20, y=0$ et $z=1 / 40$. Cette trajectoire contourne plusieurs fois des points cusps dans l'espace articulaire. L'ensemble des points forment des courbes dans l'espace articulaire et sont représentées sur la Figure 49. Ces courbes sont obtenues par l'intersection de surfaces et par discrétisation. II n'existe hélas pas de formulation paramétrée de cet ensemble de points cusps pour ce robot.

La possibilité de changer de mode d'assemblage permet à ce robot d'avoir un très grand espace de travail. En pratique, il faut tenir compte des collisions entre les segments des jambes dans la définition de l'espace de travail. Il s'agit d'un problème difficile. Dans [MD06], une méthodologie est proposée pour prendre en compte à la fois les limites articulaires, les singularités et les collisions internes pour caractériser l'espace de travail.

\subsection{Cas d'un robot parallèle cuspidal sans point cusp}

Pour les robots parallèles comme pour les robots sériels, la présence d'un point cusp est une condition suffisante pour qu'il existe des trajectoires non-singulière de changement de mode d'assemblage. Cette condition n'est pourtant pas nécessaire comme le montre l'exemple suivant.

\section{Exemple 11 : Un robot cuspidal sans cusp}

Dans [CCW14], on montre un changement de mode d'assemblage non-singulier pour un robot ne comportant pas de point cusp. Ce robot, issu du robot 3-RPR, possède une base dont la position du point $A_{3}$ varie en fonction de l'orientation de la jambe $\left(A_{1} B_{1}\right)$ (Figure 50$)$. La plate-forme mobile est un triangle rectangle formé par les points $\left(B_{1}, B_{2}, B_{3}\right)$. Deux des trois jambes sont de type

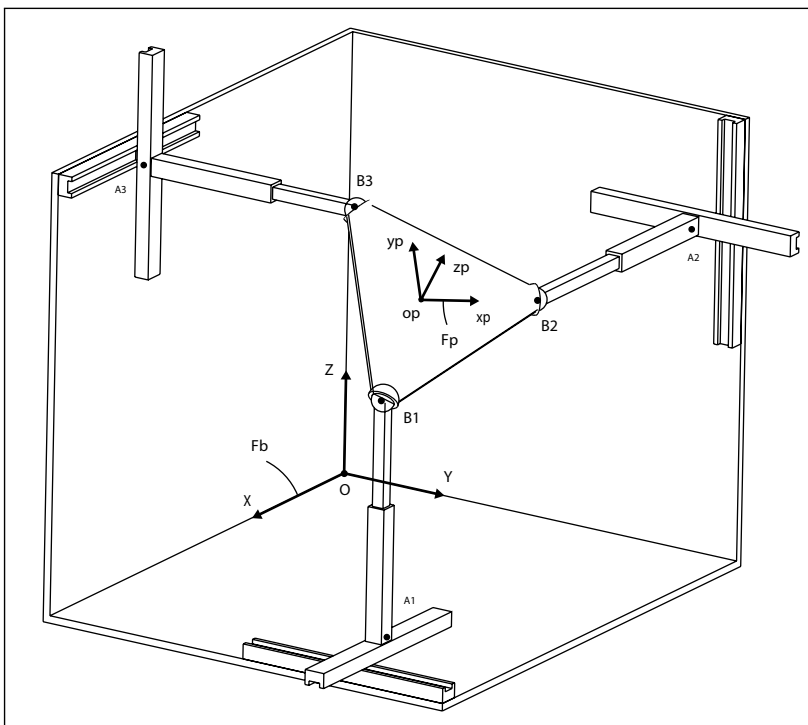

Figure 47 - Robot parallèle 3-PPPS à 6 degrés de liberté

RPR avec les articulations prismatiques actionnées $\left(r_{2}, r_{3}\right)$. Pour l'autre jambe (en rouge sur la figure $50)$, la liaison prismatique est remplacée par une barre de longueur fixe $r_{1}$. Le robot n'a donc que deux degrés de liberté. Les variables de sortie définissant l'espace de travail sont les deux angles $\theta$ et $\psi$ montrés sur la figure.

Les équations de contraintes de ce robot sont :

$$
\begin{aligned}
r_{2}^{2} & =\left(r_{1}-b_{A} \cos \theta-b_{B} \cos \psi\right)^{2} \\
& +\left(b_{A} \sin \theta+b_{B} \sin \psi\right)^{2}, \\
r_{3}^{2} & =\left(r_{1}-h_{A} \sin 2 \theta-h_{B} \sin \psi\right)^{2} \\
& +\left(h_{A} \cos 2 \theta+h_{B} \cos \psi\right)^{2} .
\end{aligned}
$$

Même si cette architecture a été créée spécifiquement pour mettre en évidence un comportement cuspidal sans cusp, elle est réalisable car il est possible d'imaginer un mécanisme de multiplication d'angle permettant de relier l'angle $\theta$ avec $\pi / 2-\theta$. Une trajectoire de changement de mode d'assemblage est représentée sur la figure 51 dans l'espace de travail et sur la figure 52 dans l'espace articulaire pour $l_{2}=1000$ et $l_{3}=800$ entre deux modes d'assemblage : $[\theta=-1.66, \psi=-0.21]$ et $[\theta=1.47, \psi=3.07]$ pour $r_{1}=30, b_{A}=10$, $h_{A}=5, b_{B}=1$ et $h_{B}=2$.

On voit que, contrairement aux robots cuspidaux ayant des points cusps, les surfaces caractéristiques ne sont jamais tangentes aux lieux des singularités mais les coupent orthogonalement. Le seul point commun avec les robots cuspidaux à points cusps est que la trajectoire de changement de mode d'assemblage traverse une région où le nombre de modes d'assemblage passe de 4 à 2 . 

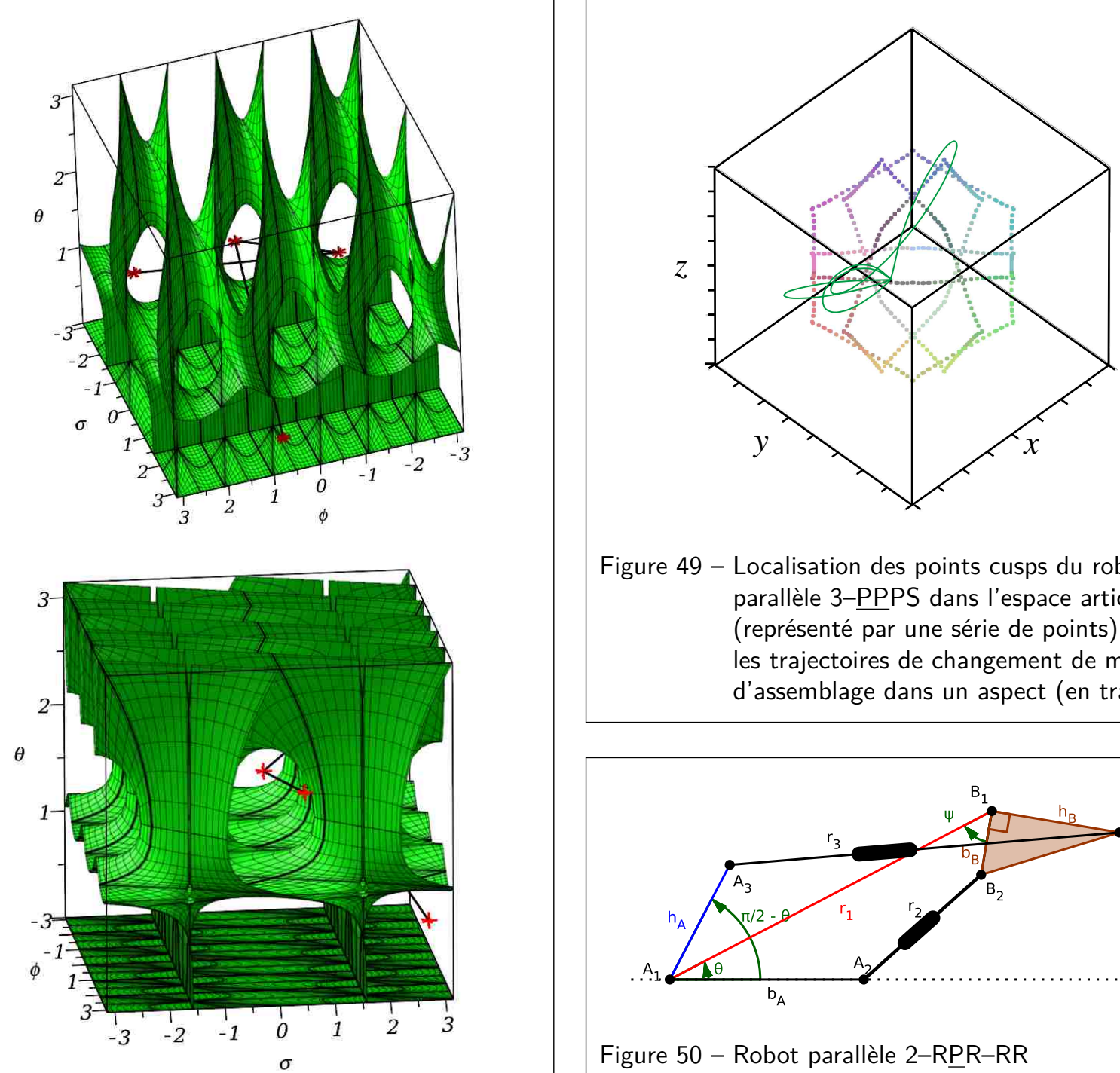

Figure 49 - Localisation des points cusps du robot parallèle 3-PPPS dans l'espace articulaire (représenté par une série de points) avec les trajectoires de changement de mode d'assemblage dans un aspect (en trait fin)

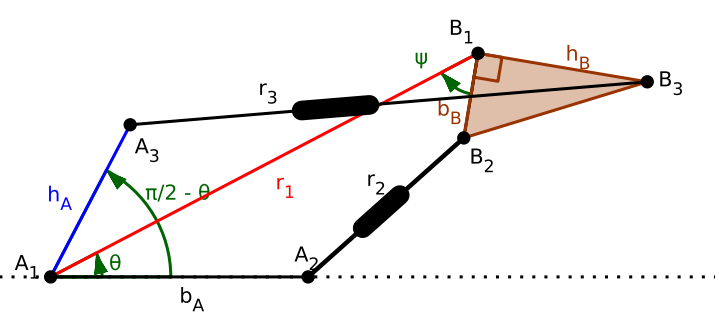

Figure 50 - Robot parallèle 2-R $\underline{P} R-R R$

Figure 48 - Exemple de trajectoire de changement de mode d'assemblage dans l'espace de travail pour le robot parallèle 3-PPPS

A retenir : Pour les robots parallèles étudiés dans cet article (n'admettant qu'une seule solution géométrique inverse), les rôles de l'espace articulaire et de travail sont inversés. Un robot parallèle est dit cuspidal lorsqu'il peut changer de mode d'assemblage, c'est-à-dire de solution géométrique directe, sans passer par une singularité. La présence de points cusp dans l'espace articulaire d'un robot parallèle indique que celui-ci est cuspidal mais la réciproque est fausse : on a pu mettre en évidence un robot parallèle cuspidal qui ne présente pas de point cusp. Les aspects sont définis dans l'espace de travail comme les domaines sans singularité les plus grands. II y a plusieurs modes d'assemblage dans un même aspect d'un robot parallèle cuspidal. Les domaines d'unicité sont définis à partir des surfaces caractéristiques dans l'espace de travail. Ces domaines permettent de garantir que le robot peut exécuter des trajectoire sans jamais changer de mode d'assemblage. Cette garantie est importante car la commande repose sur les mesures de capteur montés sur les articulations motorisés et qui ne sont pas suffisants pour définir le mode d'assemblage courant. 


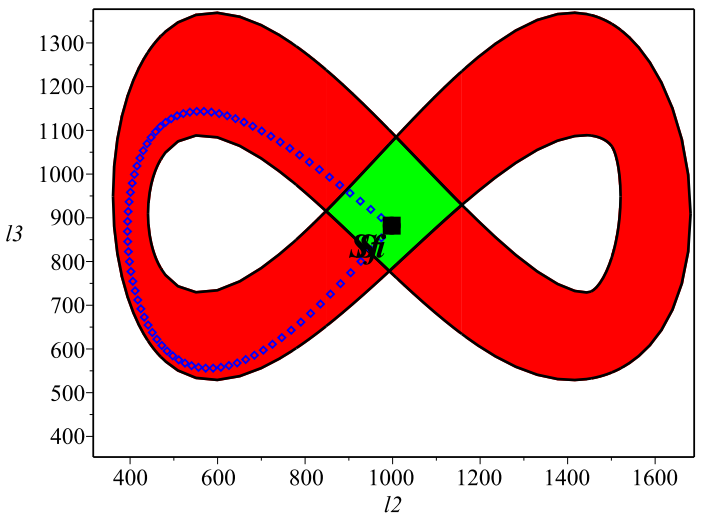

Figure 52 - Espace articulaire du robot parallèle 2-RPR-RR et trajectoire de changement de mode d'assemblage

\section{Conclusion}

Cet article s'est proposé de faire le point sur les robots dits cuspidaux, qui ont la propriété de pouvoir changer de solution du modèle géométrique inverse (robots sériels) ou direct (robots parallèles) sans passer par une singularité. Cette propriété est assez peu abordée dans la littérature. Un robot sériel (resp. parallèle) présentant au moins un point cusp dans son espace de travail (resp. dans son espace articulaire) est cuspidal mais la réciproque n'est pas toujours vraie. Particulièrement difficile à étudier en raison de la complexité des équations mises en oeuvre, l'analyse de cuspidalité nécessite le recours à des outils algébriques sophistiqués. Ces outils s'appuient en particulier sur les bases de Gröbner et la décomposition cylindrique algébrique [Mor+10] et ont été intégrés dans la librairie Siropa disponible dans le logiciel Maple [Cha+19]. L'étude de cuspidalité est très importante pour la recherche de morphologies alternatives de robots. On a vu que la propriété de cuspidalité complique l'analyse de trajectoires et n'est finalement pas souhaitable pour un robot sériel. Les architectures cinématiques des robots industriels sériels sont très peu variées et obéissent à des particularités géométriques (axes parallèles et/ou concourants) qui les rendent non cuspidales. Or, si l'on cherche à s'affranchir de ces règles pour concevoir un robot d'architecture innovante, on a toutes les chances d'obtenir un robot cuspidal. On a donné dans cet article des conditions et des règles pour qu'un robot sériel à $3 \mathrm{ddls}$ soit cuspidal ou non. Le cas des robots $6 \mathrm{R}$ reste encore un sujet de recherche ouvert. II est au coeur du projet FranceAutriche "ECARP" en cours au moment de la rédaction de cet article (https://ecarp.lip6.fr/).

Dans la dernière partie de l'article, on a évoqué le cas des robots parallèles. Peu de résultats existent pour ces robots qui sont encore très peu répandus dans l'industrie. Leur propriété de cuspidalité est très différente par rapport aux robots sériels. Loin de nuire à la planification de trajectoire, elle peut même augmenter la taille des régions t-parcourables. La principale difficulté des robots parallèles cuspidaux réside dans l'identification du mode d'assemblage dans lequel se trouve le robot à chaque instant, qui nécessite le recours à des capteurs supplémentaires ou le calcul et l'exploitation des domaines d'unicité qui garantissent de garder toujours le même mode d'assemblage, au prix d'une réduction de l'espace de travail.

Pour les robots sériels comme pour les robots parallèles, de nombreux travaux restent à réaliser pour généraliser les résultats obtenus aux robots à 6 degrés de liberté. Des outils de visualisation, de nouvelles méthodes de calcul qui mixent le calcul formel avec les méthodes numériques sont à développer. Enfin, on a vu dans cet article le rôle crucial joué par des points singuliers particuliers, les points cusps. L'impact des points cusps sur la précision et sur la commande reste à étudier avec la réalisation de démonstrateurs qui utiliseraient ces propriétés. Un point cusp est toujours une condition suffisante de cuspidalité du robot. En revanche, on a vu un exemple de robot cuspidal qui n'a pas de point cusp. Ce robot est un robot parallèle très particulier qui a été imaginé pour qu'il possède cette propriété. Aucun autre exemple de robot sériel ou parallèle n'a pu être identifié qui a la même caractéristique. Pour les robots sériels orthogonaux de type $3 R$, on a pu montrer que l'existence de points cusp est aussi une condition nécessaire. II est très probable qu'il en soit de même pour d'autres familles de robots sériels et parallèles, cette question restant encore ouverte.

\section{Glossaire}

Degré de liberté (ddl); degree of freedom (dof)

Nombre de coordonnées généralisées indépendantes requises pour définir entièrement la configuration d'un système à chaque instant (définition IFToMM).

Articulation rotoïde ; Revolute joint

Couple rotoïde (noté R) également appelé liaison pivot, autorisant une rotation autour d'un axe.

Articulation prismatique ; Prismatic joint

Couple prismatique (noté $P$ ) également appelé liaison glissière, autorisant une translation le long d'un axe.

Modes d'assemblage ; Assembly modes

Pour un robot parallèle, solution du modèle géométrique direct.

Posture ; posture

Pour un robot sériel, solution du modèle géométrique inverse.

Espace de travail ; Workspace

Ensemble des positions/orientations accessibles par l'organe terminal du robot.

Espace articulaire ; Joint space

Ensemble des positions articulaires motorisées.

Point cusp ; Cusp point

Point de rebroussement, pour désigner un type particulier de point singulier sur une courbe, où celle-ci possède une seule demi-tangente en ce point.

Robots analytiques ; Analytical robots 
Robot dont les solutions du modèle géométrique direct sont obtenues par la résolution d'équations de degrés 2, 3 ou 4 pour lesquelles il existe des racines explicites. Triangles semblables ; Similar triangles

Deux triangles sont dits semblables lorsque leurs cotés sont proportionnels 


\section{Robots cuspidaux : théorie et applications}

par Philippe WENGER

Directeur de Rercherche CNRS

Laboratoire des Sciences du Numérique de

Nantes, UMR CNRS 6004, Nantes, France

par Damien CHABLAT

Directeur de Rercherche CNRS

Laboratoire des Sciences du Numérique de

Nantes, UMR CNRS 6004, Nantes, France

\section{References}

[Bai04] Maher BAILI. "Analyse et classification de manipulateurs $3 \mathrm{R}$ à axes orthogonaux". Thèse de doct. Nantes, 2004 (cf. p. $5,8,12)$.

[BL86] Paul Borrel et Alain LiÉGeois. "A study of multiple manipulator inverse kinematic solutions with applications to trajectory planning and workspace determination". In : Proceedings. 1986 IEEE International Conference on Robotics and Automation. T. 3. IEEE. 1986, p. 11801185 (cf. p. 3, 4, 8).

[BWC04] Maher Baili, Philippe Wenger et Damien Chablat. "A classification of 3R orthogonal manipulators by the topology of their workspace". In : IEEE International Conference on Robotics and Automation, 2004. Proceedings. ICRA'04. 2004. T. 2. IEEE. 2004, p. 1933-1938 (cf. p. 3, 5).

[BZG02] IA Bonev, D Zlatanov et CM Gosselin. "Advantages of the modified Euler angles in the design and control of PKMs". In : 2002 Parallel Kinematic Machines International Conference. 2002, p. 171-188 (cf. p. 24).

[CB15] Olivier COMPANY et Sébastien BRIOT. "Les robots parallèles". In : Techniques de l'ingénieur Conception, modélisation et commande en robotique base documentaire : TIB398DUO.ref. article : s7768 (2015) (cf. p. 3, 4).

[CCW14] Michel Coste, Damien Chablat et Philippe Wenger. "Nonsingular change of assembly mode without any cusp". In : Advances in robot kinematics. Springer, 2014, p. 105-112 (cf. p. 24).
[CDS20] Jose CApco, Mohab Safey El Din et Josef Sснісно. "Robots, computer algebra and eight connected components". In : Proceedings of the 45th International Symposium on Symbolic and Algebraic Computation. 2020, p. 62-69 (cf. p. 14).

[Cha+] Damien Chablat et al. "Workspace analysis in the design parameter space of a 2-DOF spherical parallel mechanism for a prescribed workspace: Application to the otologic surgery". In : Mechanism and Machine Theory 157 (), p. 104224 (cf. p. 18).

[Cha+19] Damien Chablat et al. "Using Maple to analyse parallel robots". In : Maple Conference. Springer. 2019, p. 50-64 (cf. p. 11, 18, 26).

[Cha+20] Damien Chablat et al. "Joint space and workspace analysis of a 2-DOF Spherical Parallel Mechanism". In : European Conference on Mechanism Science. Springer. 2020, p. 181-188 (cf. p. 23).

[Cha+21] Damien Chablat et al. “Workspace analysis in the design parameter space of a 2-DOF spherical parallel mechanism for a prescribed workspace: Application to the otologic surgery". In : Mechanism and Machine Theory 157 (2021), p. 104224 (cf. p. 23).

[Cha98] Damien Chablat. "Domaines d'unicité et parcourabilité pour les manipulateurs pleinement parallèles". Thèse de doct. Ecole Centrale de Nantes (ECN), 1998 (cf. p. 5, 20).

[CJR92] Gosselin CLÉMENT, Sefrioui Jaouad et MJ Richard. “Solutions polynomials au probléme de la cinématique des manipulateurs paralléles plans á trois de gré de liberté". In : Mech. Mach. Theory 27 (1992), p. 107-119 (cf. p. 17).

[CMW11] Damien Chablat, Guillaume Moroz et Philippe Wenger. "Uniqueness domains and non singular assembly mode changing trajectories". In : 2011 IEEE International Conference on Robotics and Automation. IEEE. 2011, p. 3946-3951 (cf. p. 20, 21).

[CR04] S. Corvez et F. Rouillier. "Using computer algebra tools to classify serial manipulators". In : Automated Deduction in Geometry, Lectures Notes in Computer Science. 2004, p. 31-43 (cf. p. 11).

[CW98] Damien Chablat et Philippe Wenger. "Working modes and aspects in fully parallel manipulators". In : Proceedings. 1998 IEEE International Conference on Robotics and Automation (Cat. No. 98CH36146). T. 3. IEEE. 1998, p. 19641969 (cf. p. 3, 16). 
[CW99] Damien Chablat et Philippe Wenger. "On the characterization of the regions of feasible trajectories in the workspace of parallel manipulators". In : IFTOMM. 1999 , p. 1-6 (cf. p. 20).

[CWC11] Michel Coste, Philippe Wenger et Damien Chablat. "Singular surfaces and cusps in symmetric planar 3-RPR manipulators". In : 2011 IEEE/RSJ International Conference on Intelligent Robots and Systems. IEEE. 2011, p. 1453-1458 (cf. p. 20).

[CWC12] Stéphane CAro, Philippe Wenger et Damien Chablat. "Non-singular assembly mode changing trajectories of a 6-DOF parallel robot". In : t. 45035. American Society of Mechanical Engineers. 2012, p. 1245-1254 (cf. p. 23).

[Deb+01] Thomas Debus et al. "Cooperative Human and Machine Perception in Teleoperated Assembly". In : Experimental Robotics VII. Sous la dir. de Daniela Rus et Sanjiv SingH. Berlin, Heidelberg : Springer Berlin Heidelberg, 2001, p. 5160. ISBN : 978-3-540-45118-1 (cf. p. 14).

[El 96] Jaouad El OMri. "Analyse géométrique et cinématique des mécanismes de type manipulateur". Thèse de doct. Nantes, 1996 (cf. p. 5, 7, 10, 11, 14).

[EW95] J El OMri et P Wenger. "How to recognize simply a non-singular posture changing 3-DOF manipulator". In : Proc. 7th Int. Conf. on Advanced Robotics. 1995 , p. 215-222 (cf. p. 3, 5, 7).

[IP92] C Innocenti et V Parenti-Castelli. “Direct kinematics of the 6-4 fully parallel manipulator with position and orientation uncoupled". In : Robotic systems. Springer, 1992, p. 3-10 (cf. p. 18).

[IP98] C Innocenti et V Parenti-Castelli. "Singularity-free evolution from one configuration to another in serial and fully-parallel manipulators". In : ASME J. Mechanical Design 120 (1998), p. 73-99 (cf. p. 17).

[KD99] W. Khalil et E. Dombre. Modélisation, identification et commande des robots. Collection robotique. Hermes Science $\mathrm{Pu}-$ blications, 1999. ISBN : 9782746200036 (cf. p. 11, 12, 14).

[KG00] Xianwen Kong et Clément M Gosselin. "Determination of the uniqueness domains of 3-RPR planar parallel manipulators with similar platforms". In : International Design Engineering Technical Conferences and Computers and Information in Engineering Conference. T. 35173. American Society of Mechanical Engineers. 2000, p. 419-426 (cf. p. 19).
[MD06] Jean-Pierre Merlet et David Daney. "Legs interference checking of parallel robots over a given workspace or trajectory". In : Proceedings - IEEE International Conference on Robotics and Automation. Fév. 2006, p. 757-762. ISBN : 0-78039505-0 (cf. p. 24).

[MD99] P Ross McAree et R William Daniel. "An explanation of never-special assembly changing motions for 3-3 parallel manipulators". In : The International Journal of Robotics Research 18.6 (1999), p. 556-574 (cf. p. 18).

[Mer96] J-P MerLet. “Direct kinematics of planar parallel manipulators". In : Proceedings of IEEE international conference on robotics and automation. T. 4. IEEE. 1996, p. 37443749 (cf. p. 18).

[Mer97] Jean-Pierre MerLet. “Les robots parallèles. Traités de nouvelles technologiques". In : (1997) (cf. p. 17).

[Mor+10] Guillaume Moroz et al. "On the determination of cusp points of 3-RPR parallel manipulators". In : Mechanism and Machine Theory 45.11 (2010), p. 15551567 (cf. p. 18, 26).

[NBW12] Dinh Quan NguYEN, Sébastien Briot et Philippe Wenger. "Analysis of the Dynamic Performance of Serial 3R Orthogonal Manipulators". In : ASME 2012 11th Biennial Conference on Engineering Systems Design and Analysis. 2012, p. $175-184$ (cf. p. 15).

[Pfu09] Martin Pfurner. "Explicit algebraic solution of geometrically simple serial manipulators". In : Computational Kinematics. Springer, 2009, p. 167-174 (cf. p. 14).

[PI88] C.V. Parenti et C. Innocenti. "Position Analysis of Robot Manipulators : Regions and Sub-regions". In : Proceedings of Int. Conf. on Advances in Robot Kinematics. 1988, p. 150-158 (cf. p. 3, 14, 15).

[Pie68] DL Pieper. "The Kinematics of manipulators under Computer control". Thèse de doct. Standford University, 1968 (cf. p. 8,11$)$.

[WC91] Philippe Wenger et Patrick Chedmail. "Ability of a robot to travel through its free work space in an environment with obstacles". In : The International journal of robotics research 10.3 (1991), p. 214-227 (cf. p. 8).

[WC97] Philippe Wenger et Damien Chablat. "Definition sets for the direct kinematics of parallel manipulators". In : 1997 8th International Conference on Advanced Robotics. Proceedings. ICAR'97. IEEE. 1997, p. 859-864 (cf. p. 16). 
[WC98] Ph. Wenger et D. Chablat. “Workspace and Assembly Modes in Fully-Parallel Manipulators: A Descriptive Study". In : Advances in Robot Kinematics: Analysis and Control. Sous la dir. de Jadran Lenarčič et Manfred L. Husty. Dordrecht: Springer Netherlands, 1998, p. 117-126. ISBN : 978-94-015-9064-8 (cf. p. 23).

[WCZ07] Philippe Wenger, Damien Chablat et Mazen Zein. "Degeneracy study of the forward kinematics of planar 3-RPR parallel manipulators". In : Journal of Mechanical Design 129.12 (2007), p. 1-6 (cf. p. 19).

[Wen04] P. Wenger. "Uniqueness domains and regions of feasible paths for cuspidal manipulators". In : IEEE Transactions on Robotics 20(4) (2004), p. 754-750 (cf. p. 9, 10).

[Wen07] Philippe Wenger. "Cuspidal and noncuspidal robot manipulators". In : Robotica 25.6 (2007), p. 677-689. DOI : 10 . 1017/S0263574707003761 (cf. p. 3, 14).

[Wen92] Philippe Wenger. "A new general formalism for the kinematic analysis of all nonredundant manipulators". In : Proceedings 1992 IEEE International Conference on Robotics and Automation. IEEE Computer Society. 1992, p. 442-443 (cf. p. 3).

[Wen97] P. Wenger. "Design of cuspidal and noncuspidal manipulators". In : Proceedings of IEEE Int. Conf. Rob. and Aut. 1997, p. 2172-2177 (cf. p. 11).

[Whi55] H. Whitney. "On singularities of mappings of Euclidean spaces 1. Mappings of the plane into the plane". In : Annals of Mathematics 62(3) (1955), p. 374-410 (cf. p. 6).

[ZWC07a] Mazen Zein, Philippe Wenger et Damien Chablat. "A design oriented study for 3R Orthogonal Manipulators With Geometric Simplifications". In : Congrès international Conception et Modélisation des Systèmes Mécaniques. 2007, p. 1-8 (cf. p. 15).

[ZWC07b] Mazen Zein, Philippe Wenger et Damien Chablat. "Singular Curves in the Joint Space and Cusp Points of 3-RPR parallel manipulators". In : Robotica 25.arXiv: 0711.0574 (2007), p. 717-724 (cf. p. 18).

[ZWC08] Mazen Zein, Philippe Wenger et Damien Chablat. "Non-singular assembly-mode changing motions for 3-RPR parallel manipulators". In : Mechanism and Machine Theory 43.4 (2008), p. $480-490$ (cf. p. 18).

\section{À lire également dans nos bases}

Albert COLIN - Bras de manipulation et robots industriels [A9150 v1] (1995)

Alain LIÉGEOIS - Modélisation et commande des robots manipulateurs [s7730] (2000)

Olivier COMPANY, Sébastien BRIOT - Les robots parallèles [s7768] (2015)

\section{Outils logiciels}

Citer Maple et Matlab?

\section{Sites Internet}

SIROPA http:/ / siropa.gforge.inria.fr/ 